\title{
A species-specific signature residue in the PB2 subunit of the bat influenza virus polymerase restricts viral RNA synthesis.
}

Saptarshi Banerjee ${ }^{a}$, Aratrika De ${ }^{a}$, Nandita Kedia ${ }^{a}$, Wang Linfa ${ }^{b}$, Arindam Mondal ${ }^{a} \#$

aSchool of Bioscience, Indian Institute of Technology Kharagpur, Kharagpur, India

${ }^{\mathrm{b}}$ Duke-NUS Medical School, Singapore City, Singapore

\#Address correspondence to Arindam Mondal, arindam.mondal@iitkgp.ac.in

Word count for abstract and text: 5734

\section{Abstract}

Bat influenza A viruses (H17N10 and H18N11) are genetically distant from conventional influenza A viruses and replicates poorly in non-bat hosts species. However, the reason behind the lower replication fitness of these viruses are yet to be elucidated. In this work, we have identified species-specific signature residues, present in viral PB2 protein, which is a major determinant of polymerase fitness in human, avian and bat cell lines. Through extensive sequence and structural comparison between the bat and non-bat influenza virus RNA polymerases, we have identified a previously uncharacterized PB2-282 residue, which is serine in bat virus PB2 protein but harbours highly conserved glutamic acid in conventional influenza A viruses. Introduction of these bat specific signatures in 
22 the polymerase of a human adapted strain of influenza $\mathrm{A} / \mathrm{H} 1 \mathrm{~N} 1$ virus drastically reduces

23 its polymerase activity and replication efficiency in cell lines of human, bat and canine

24 origin. In contrast, introduction of the human specific signatures in bat virus PB2

25 (H17N10), significantly enhances its function in the context of a chimeric RNA

26 polymerase. Interestingly, the PB2-282 resides within an evolutionary conserved "S-E-S"

27 motif present across different genera of influenza viruses but is replaced with a "S-S-T"

28 motif in bat influenza viruses, indicating that this $E$ to $S$ transition may serve as a species-

29 specific adaptation signature that modulates the activity of bat virus polymerase in other

30 host species.

\section{Importance}

32 Recent isolation of influenza A like viruses (H17N10 and H18N11) in bats raised

33 concerns about their potential of zoonotic transmission in human. Here we present

34 species-specific signature residues present in the bat influenza virus polymerase, which

35 may act as critical modulators of bat virus propagation in non-bat host species. We

36 utilize bioinformatics based comparative analysis followed by functional screening in

37 order to identify the PB2-282 ${ }^{\text {nd }}$ position, which harbors a highly conserved glutamic acid

38 in conventional influenza A viruses, but contains an unusual serine in case of bat

39 influenza viruses. Human adapted polymerase, harboring bat specific signature (PB2-

40 S282) performs poorly, while bat PB2 protein harboring human specific signature (PB2-

41 E282) shows increased fitness in human cells. Together, our data identifies novel

42 species-specific signatures present within the influenza virus polymerase that may

43 serve as a key factor in the adaptation of influenza viruses from bat to non-bat host

44 species and vice versa. 
46 Keywords- Host adaptation, Bat Influenza A virus, Avian influenza virus, RNA dependent

47 RNA polymerase, Ribonucleoprotein complex,

\section{Introduction}

Influenza A viruses are segmented negative sense RNA viruses that cause respiratory

51 infections in humans and wide range of other animals including, but not limited to, birds,

52 pigs, dogs, cats, horses and seals (1). Wild aquatic birds are the natural reservoirs for

53 these viruses, which through continuous adaptation in their genetic architecture acquires

54 ability to infect new host species. Such adaptation often time results in a new event of

55 zoonotic infection leading to the expansion in the repository of human infecting influenza

56 A viruses (1). For successful zoonoses, influenza viruses have to conquer two barriers

57 imposed by the host; first, successful recognition of the cell surface receptors by viral HA

58 protein to mediate entry (2), and second, species specific adaptation of the viral RNA

59 dependent RNA polymerase (RdRp)(3), majorly governed by its PB2 subunit. The PB2

60 protein of avian viruses contains a glutamic acid residue at the $627^{\text {th }}$ position while for the

61 human adapted viruses, the same position harbors a lysine. The polymerase containing

62 avian type signature residue in PB2 is fully functional in avian cells, but shows severe

63 attenuation in human cells $(4,5)$. Other mutations within the 627 domain of PB2 have

64 also been reported to act as species specific adaptation sites that helps the avian viruses

65 to establish successful infection in humans/ mammals (6). 
During 2012 and 2013, genetic traces of two new lineages of influenza A viruses were isolated from the little yellow-shouldered bat (Sturnira lilium), in Guatemala, and the flatfaced fruit-eating bat (Artibeus planirostris), in Peru respectively $(7,8)$. According to the phylogenetic analyses, these two viruses shows significant divergence in their genetic architecture from classical influenza A viruses, classified as two new subtypes, the $\mathrm{H} 17 \mathrm{~N} 10$ and $\mathrm{H} 18 \mathrm{~N} 11$. The $\mathrm{H} 17$ and $\mathrm{H} 18$ proteins do not recognize the canonical sialic acid receptors $(8,9)$, while the $\mathrm{N} 10$ and N11 lacks sialidase activity (10), suggesting that bat influenza viruses utilize distinct mechanism for entry into the host cells as compared to the classical influenza A viruses. Recent advancements with bat influenza viruses further substantiated this notion through identification of MHC class II (MHCII) as bonafied receptors for bat influenza viruses $(11,12)$. This also has raised the concerns of epizootic and zoonotic transmission of these viruses, given the constitutive expression of $\mathrm{MHCII}$ molecules in wide variety of tissues in human, pigs, chicken and wide range of other animals (11).

Polymerases from human or avian origin influenza viruses have shown to quickly adapt

81 in bat cell lines (13). However, adaptability of the bat influenza virus polymerase in avian,

82 human, or in other mammalian hosts has yet to be extensively investigated. Chimeric bat 83 viruses that express surface antigens (HA and NA) of classical influenza viruses 84 (influenza A/H1N1/PR8 or A/H7N7/SC35M) but internal proteins from the bat influenza 85 virus (H17N10/Guatemala/164/2009) replicates poorly in cell culture and in infected animals compared to the H1N1 (PR8) or H7N7 (SC35M) viruses (14, 15). Additionally,

87 recombinant bat virus polymerase reconstituted in human lung epithelial cells shows 88 limited activity in a reporter-based polymerase activity assay (7). Together, these 
evidences suggest that bat influenza virus polymerases are severely restricted in non-bat hosts, possibly due to the higher genetic diversity of the internal genes (16) that accommodate unique signature residues in the individual subunit proteins, PB2, PB1 and PA. In this regard it should be noted that the bat virus PB2 protein harbors an unusual serine residue at the $627^{\text {th }}$ position instead of highly conserved glutamic acid or lysine as in case of avian or human adapted viruses respectively.

In this work, we characterized unique molecular signatures present in the bat influenza virus PB2 protein that could be responsible for the compromised fitness of the polymerase in non-bat hosts. Considering the PB2-S627 as signature for the bat virus polymerase, we tried to identify more such positions in the PB2 protein, which harbors either serine or threonine in the bat virus but contains highly conserved glutamic acid or aspartic acid in the non-bat IAV's. We have identified the Serine at 282 position as a key signature residue, responsible for restricted activity of the bat virus polymerase, in comparison to the human or avian adapted polymerases harboring a glutamic acid residue at the same position. Replacing the glutamic acid either with alanine, lysine or with bat specific serine residue severely affects the RNA synthesis activity of the human virus polymerase when reconstituted through transient transfection. Furthermore, recombinant human viruses with alanine or bat specific serine at 282 position is severely restricted in cell lines derived from human, bat and canine, establishing the importance of the glutamic acid at this position. This is further supported by the fact that partially humanized H17N10 PB2, harboring glutamic acid at 282 position boosts polymerase activity several folds compared

110 to its wildtype version harboring serine at the same position. Together, our work identified 111 a key molecular signature in bat influenza virus PB2 protein which is responsible for its 
112 restricted activity in non bat host species and may serve as a potential site of adaptation

113 in order to acquire improve fitness in other hosts including human.

114 Results:

115 Identification of the bat specific signatures responsible for the restricted activity of

116 the polymerase in non-bat host.

117 In order to identify the species-specific signature residues in the bat virus polymerase we

118 focused upon the PB2 subunit due to its critical role in determining the species specificity

119 of influenza A viruses $(4,5)$. Throughout this study the influenza A/H1N1/WSN/1933

120 strain was used as a model for the human adapted virus while the influenza

$121 \mathrm{~A} / \mathrm{H} 17 \mathrm{~N} 10 / \mathrm{Guatemala/60}$ strain was used as a representative for the bat virus. First, we

122 replaced the human-specific lysine at the $627^{\text {th }}$ position with the bat-specific serine

123 residue in order to ascertain its impact upon the activity of the human virus polymerase

124 in human (HEK293T) and avian (DF1) cells. Luciferase based reporter RNPs were 125 reconstituted through transient transfection of a genome sense reporter RNA template,

126 polymerase subunits (PB1, PB2 and PA) and NP expressing plasmids derived from the

$127 \mathrm{~A} / \mathrm{H} 1 \mathrm{~N} 1 / \mathrm{WSN} / 1933$ strain as described previously (Mondal et al, 2015). Different variants

128 of PB2 harboring the human signature lysine $(\mathrm{K})$, avian signature glutamic acid $(\mathrm{E})$ or bat

129 signature serine $(\mathrm{S})$ residues at the $627^{\text {th }}$ position were used to reconstitute the 130 polymerase (Fig1.A). The polymerase with PB2-627K shows high reporter activity in both

131 the cell lines while the polymerase with PB2-627E was selectively attenuated in human

132 cells as expected $(4,5,18)$, hence validating the efficiency of our luciferase-based assay

133 system in determining the species-specific fitness of the polymerase (Fig1.B).

134 Interestingly, the polymerase with PB2-627S still remained attenuated, although to a 
135 lesser extent than PB2-627E, in human cells but was fully functional in chicken cells

136 (Fig1.B). Different PB2 variants shows comparable expression as evidenced from the

137 western blot analysis (Fig1.B). These data suggest that the PB2-627S may serve as one

138 of the key determinants for species specific restriction of the bat virus polymerase in non-

139 bat host species, specifically in human.

140 Encouraged by this finding, we focused our study towards identification of additional

141 signature residues, present in the bat influenza virus PB2 protein, which may regulate the

142 activity of viral RNA polymerase in a species-specific manner. Using the PB2-627S as a

143 reference, we tried to identify specific positions throughout the primary sequence of PB2

144 protein, which harbors either serine or threonine in the bat virus polymerase but

145 represents highly conserved glutamic acid or aspartic acid in the conventional influenza

146 viruses of either human or avian origin. Through extensive alignment of the bat and not

147 bat influenza A virus PB2 sequences we have been able to identify total seven such amino

148 acid residues $(60,282,390,472,671,678,681)$, which were then further screened based

149 upon their solvent accessibility in the atomic structure of the polymerase $(19,20)$. Finally,

150 we focused upon five amino acid residues, 282, 390, 472, 678, 681 that are completely

151 surface exposed (Fig2.A, table 1) in the heterotrimeric polymerase (in analogy with the

152 PB2-627 position), harbor serine in bat virus PB2 protein but are occupied by highly

153 conserved aspartic acid $(390,678)$ or glutamic acid $(282,472,681)$ in the non-bat IAV's

154 (Table1 and Fig1A). Residue number 60 and 671 were not included in the study as they

155 are not completely surface exposed in the heterotrimeric polymerase rather reside within

156 the PB2-PB1 and PB2-PB1-PA interaction interface respectively. We speculated that 
157 some of these residues may serve as a determining factor in regulating the bat virus 158 polymerase activity in bat and (or) non-bat host species.

In order to test this, we first investigated the functional significance of the identified amino acid residues in the context of the human adapted polymerase. For this purpose,

161 we have substituted each of the aspartic acid or glutamic acid residues with alanine in 162 order to generate a panel of the mutant PB2 proteins. Luciferase based reporter RNPs were reconstituted either with wild type or with mutant PB2 proteins along with other RNP components as described earlier. Polymerase reconstituted with the wildtype PB2 protein showed high level of reporter activity in HEK293T cells while mutant PB2 proteins supported polymerase activity to various extents (Fig 2.B). The PB2 D472A and E678A

167 mutants supported polymerase activity comparable to the wild type protein suggesting 168 that these residues are not critical for viral RNA synthesis. The D390A and E681A 169 mutants showed only modest defects reducing polymerase activity only by $40 \%$.

170 Interestingly, the E282A mutation severely attenuated the polymerase, supporting only $17120 \%$ reporter activity compared to the wildtype PB2. These data suggest that the glutamic 172 acid at 282 position of PB2 is critical in supporting activity of the human adapted 173 polymerase in human cells. Western blot analysis of the E282A and other mutant PB2 174 proteins showed expression levels comparable to the wild type protein, hence confirming 175 that the defect in RNA synthesis results from the suboptimal activity of these proteins and 176 not from their altered abundance in cells. 
178 Recombinant influenza A/H1N1/WSN viruses harboring bat specific signature residues at the 627 and 282 position of PB2 shows reduced fitness in bat and not bat host species.

181

182

Highly conserved PB2-K627 and PB2-E282 residues are functionally important in the context of human virus polymerase suggesting that the bat specific serine residues at corresponding positions may alter the polymerase activity and hence interfere with virus propagation in a species-specific manner. Hence, we investigated the impact of the bat specific serine residues at the $627^{\text {th }}$ and $282^{\text {nd }}$ positions of PB2 in the context of the propagation of human adapted viruses. The genetic background of the influenza A/H1N1/WSN/1933 strains was used and K627S, E282A and E282S mutations were introduced in the PB2 open reading frame in order to generate recombinant viruses using the plasmid based reverse genetics as described earlier(18). Initially rescued viruses were amplified in MDCK cells for three consecutive passages, then titrated using plaque assay and finally confirmed the presence of the mutations in the PB2 ORF through sanger sequencing. While the PB2-627S virus shows plaque sizes comparable to the wild type virus, PB2-282A and PB2-282S viruses showed significantly smaller plaque sizes indicating possible defect in the replication of the mutant viruses (Fig 3A). Interestingly, the E282S mutant virus incorporated a conservative leucine to valine replacement at the adjacent 281 position which occurred consistently for the viruses rescued in two independent experiments. The reverse genetics plasmid harboring the PB2 open reading frame showed no such change in its nucleotide sequence suggesting that the virus spontaneously incorporated L281V mutation in its genomic RNA sequence. It should be noted that the E282 residue is conserved not only for influenza A, but also for influenza 
201 B and C viruses, where this glutamic acid residue is preceded by the highly conserved

202 valine as observed in the case of our E282S/L281V mutant virus. Hence, we moved

203 forward with the mutant viruses to investigate their ability to propagate in cell lines from

204 various host species. For simplicity, we designate the E282S/L281V mutant virus as

205 E282S virus for the rest of the manuscript.

206 First, we have evaluated the fitness of the mutant PB2 viruses by performing multicycle 207 replication kinetics in Madin-Darby Canine Kidney (MDCK) cells. The wildtype virus 208 replicated to high levels reaching titers more than $10^{7}$ by 48 hours post infection, while the PB2-K627S virus was severely attenuated showing up to 1000-fold reduction in viral 210 titer throughout the time course (Fig3.A). This clearly reflects that introduction of the bat 211 specific residue in the human polymerase severely restricts its activity in cells of 212 mammalian origin, which is consistent of the results demonstrated with others strains of 213 influenza $\mathrm{A} / \mathrm{H} 1 \mathrm{~N} 1$ virus (21). Introduction of either alanine or the bat specific serine 214 residue at the 282-position showed similar attenuation with a 100-fold decrease in the 215 titer with respect to wildtype virus (Fig3.A). Interestingly, the PB2-E282S virus showed 216 higher replication fitness compared to the PB2-K627S virus in MDCK cells. To have a 217 better insight into the specie-specific fitness of human influenza virus that could be 218 modulated by the bat specific serine residues at the PB2-627 and PB2-282 positions, we 219 next evaluated the replicability of the mutant viruses in cell lines of human and bat origin. 220 We have used human lung epithelial carcinoma cells (A549) and Pteropus alecto kidney 221 (PaKi) cells as model cell lines of the respective host species. As described earlier, the

222 PaKi cells support influenza virus infection and re-assortment (22), which makes them 223 suitable for monitoring replication of the human viruses harboring bat specific signatures 
224 in their replication machinery. In fact, both wildtype and mutant viruses replicated to

225 higher extents in PaKi cells compared to the A549 or MDCK cells showing high

226 susceptibility and permissiveness of these cells towards H1N1 virus infection (Fig3.C). In

227 A549 cells, replication efficiency of the wild type and mutant viruses showed trends similar

228 to what was observed in MDCK cells. The PB2-K627S showed up to 100-fold attenuation

229 followed by the PB2-E282S showing around 10-fold reduction in viral titers as compared

230 to the wild type virus (Fig3.B). Interestingly, both the mutant viruses showed similar

231 replication fitness in PaKi cells, with more than 100-fold reduction in virus titer as

232 compared to the wild type one (Fig3.C). Together our data suggest introduction of the bat

233 specific serine residues at the critical 627 and 282 positions of PB2 can significantly

234 restrict the replication of the prototypic WSN strain of human influenza virus in both bat

235 and non-bat host species. Based upon the sequence and structural conservation of the

236 above-mentioned residues, it is likely that similar phenotypes might be observed for other

237 conventional influenza viruses as well.

238 A highly conserved " $S_{279}-E_{282}-S_{286}$ " motif within the mid-link regions of the PB2 protein is crucial for optimum fitness of the polymerase in human and avian cells.

240 The E282 resides within the "mid-link" region of PB2, which connects the N terminal and 241 cap binding domains. As revealed by different atomic structures of the polymerase, the 242 "mid link" region along with the "627-linker", together forms the stock upon which the cap 243 binding domain and the 627 domain can undergo structural reorganization in order to 244 transition between the transcriptionally active and replicative forms $(19,20)$. The mid link 245 region consists of four $\alpha$ helices ( $\alpha 14-\alpha 17)$, two of which face towards the core of the 246 polymerase, while the other two are solvent exposed. The E282 resides on the second 
247 turn of the $\alpha 15$ helix flanked by two other serine residues in the adjacent turns, all three

248 facing towards the solvent (Fig4.A). Due to this structural feature the three residues,

249 S279, E282 and S286, constitute a unique motif, which is completely conserved across

250 the PB2 sequences derived from the conventional influenza A viruses. Furthermore,

251 extensive structural and sequence alignment studies revealed that this S-E-S motif is also

252 conserved in influenza C viruses and partially conserved in influenza B and D viruses

253 (Fig4.A, B). Interestingly, the bat influenza A virus PB2 harbors a serine at the 282

254 position and a threonine at 286 , which results in an altered $\mathrm{S}_{279} \mathrm{~S}_{282} \mathrm{~T}_{286}$ motif with the 255 same structural feature (Fig4.B).

257 Based upon the importance of the E282 position in supporting viral RNA synthesis and virus replication and the high conservation of the "S-E-S" motif across different influenza

259 virus PB2 protein, we next evaluated the importance of this motif in supporting polymerase activity in HEK293T and DF1 cells. For this purpose, we generated a series of mutant PB2 proteins where individual amino acids of the S-E-S motif were substituted either with alanine, serine or lysine and tested their ability to support viral RNA synthesis using the reporter-based polymerase activity assay (Fig4.C). Substitution of both of the peripheral serine residues with alanine resulted in $40 \%$ decrease in reporter activity, while substitution of the central glutamic acid showed around $70-80 \%$ decrease (Fig4.C), as

266 also evidenced earlier (Fig2.B). A triple alanine mutant, substituting the entire S-E-S motif 267 resulted in complete abrogation of RNA synthesis, pointing towards the importance of the 268 entire motif in supporting the activity of viral RNA polymerase. It is interesting to note that 269 introduction of bat specific serine residue at 282 position and hence reconstituting the bat 
270 like "SSS" motif resulted in around 60-70\% decrease in polymerase activity, further

271 emphasizing the importance of the negatively charged glutamic residue at the center of

272 the motif (Fig4.C). This is also substantiated by the fact that substitution of the negatively

273 charged glutamic acid with positively charged lysine results in complete abrogation of the

274 polymerase activity. All of the mutant proteins show expression and stability comparable

275 with the wild type PB2 protein as evidenced from the western blot analysis (Fig4.C).

276 Together our data revealed the existence of a highly conserved "S-E-S" motif present in

277 the mid link region of influenza A virus PB2 protein, which is crucial in supporting the

278 polymerase activity both in avian and human cell lines.

279

280 Extensive structural and sequence comparison between influenza A and B virus PB2 281 proteins suggest that the B-PB2 protein lacks the canonical " $S_{279}-E_{282}-S_{286}$ " motif, in spite 282 of having significant sequence similarity within the mid-link region (Fig4.B). Instead, it 283 contains two glutamic acid residues in the two subsequent turns of the alpha helix, hence constituting the "E $E_{280}-E_{284}$ " motif where the E-284 of B-PB2 perfectly aligns with the E282 of A-PB2 protein (Fig4.B). To investigate whether the conserved glutamic acid at 284 is important for influenza B polymerase activity, we have developed a firefly luciferase-

287 based influenza B polymerase activity assay using the genetic background of B/Brisbane/60/2008 strain. Influenza B polymerase with wild type PB2 supported high

289 levels of reporter activity as shown in figure 4D. Interestingly, introduction of the E284A 290 mutation in B-PB2 reduced the reporter activity by $84 \%$, suggesting indispensable role of 291 this glutamic acid in supporting polymerase activity. This data further substantiates the 292 importance of this highly conserved glutamic acid residue in the PB2 protein of influenza 
293 viruses across different genera. This also points towards the fact that absence of such an 294 important molecular signature may significantly impact efficiency of the bat influenza virus 295 PB2 protein, thereby restricting the overall activity of the bat virus polymerase in 296 comparison to the conventional influenza A or influenza B viruses.

Alteration of the "SES" motif specifically restricts the RNA synthesis ability of the polymerase RNA synthesis. Influenza viruses assemble their RNA synthesis machinery in the form of viral ribonucleoprotein complexes (RNPs), where the heterotrimeric RNA polymerase (PB1, PB2 and PA) recruits multiple copies of NP on the nascent RNA strand during its synthesis, resulting in the formation of a macromolecular RNA-protein complex of megadalton range. Hence, fruitful assembly of viral RNPs are prerequisite for subsequent rounds of RNA synthesis. To investigate the molecular mechanism by which mutations introduced in the S-E-S motif altered viral RNA synthesis, we assessed their ability to support viral RNP assembly using RNP reconstitution. Authentic viral RNPs are reconstituted in HEK293T cells by expressing the negative sense genomic RNA template, NP, PB1, PA and wild type or mutant variants of Flag tagged PB2 proteins. The efficiency of RNP formation was determined by immunoprecipitating the viral polymerase via PB2FLAG and detecting co-precipitated NP as a part of RNP complex by western blot analysis. A strong signal of NP reflects co-precipitation of large number of NP molecules 
316 of direct PB2-NP interaction. As evidenced in figure5.A, wild type PB2 efficiently co-

317 purified large portions of NP indicating efficient RNP formation. The AAA and the SKS

318 mutants completely abolished the RNP formation while the SAS mutant also showed a

319 drastic reduction. AEA and the SSS mutants showed and intermediate phenotype,

320 showing higher levels of RNP formation than SAS mutant but lower than the wild type

321 protein. Together, the ability of the PB2 mutants to support RNP assembly perfectly

322 corroborates with their ability to support viral RNA synthesis as evidenced in the reporter

323 activity assay.

324

325 Fruitful assembly of RNPs relies upon efficient binary interactions between PB2 with PB1

326 and NP. Hence, we tested the ability of the mutant PB2 proteins to participate in these

327 protein-protein interactions. Flag tagged wild type or mutant PB2 proteins were co-

328 expressed either with NP or with PB1 in HEK293T cells and the lysates containing the

329 binding partners were subjected to PB2-FLAG immunoprecipitation followed by western

330 blotting to detect the coprecipitation. To eliminate any non-specific complex formation

331 with cellular RNA, lysates were treated with high amounts of RNase A before

332 immunoprecipitation. All of the PB2 mutants showed NP and PB1 coprecipitation

333 comparable to the wildtype protein suggesting mutations in the S-E-S motif does not

334 impact the ability of PB2 to interact with other RNP associated proteins (Fig5.B \& C). We

335 have also evaluated the nuclear localization ability of PB2 mutants by overexpressing

336 them in A549 cells followed by indirect immunofluorescence assay. PB2 contains nuclear

337 localization signal which directs its importin alpha mediated nuclear import in order to

338 participate in RNP formation $(23,24)$. All of the mutant proteins showed preferential 
340 transfection in HEK293T cells (Fig.6). Based upon these data it can be predicted that 341 neither impaired protein-protein interaction nor alteration of the subcellular localization 342 was the key for the altered RNP formation ability as evidenced in case of the PB2 mutants.

Synthesis of viral genomic/ antigenomic RNA and their assembly into progeny viral RNPs

345 takes place in a concomitant fashion. Hence, any defect in viral RNA synthesis would get manifested in the form of defective RNP assembly and vice versa. To test whether PB2

347 mutants are actually defective in their ability to support viral RNA synthesis, we have performed a mini-vRNA template-based primer extension assay where the RNA synthesis activity of the polymerase could be monitored independent of RNP assembly process. The mini-vRNA templates are short RNAs (76 nucleotide long) containing viral UTRs that could be replicated and transcribed by the polymerase in the absence of NP (25). Hence, any defect in RNA synthesis could solely be attributed to the defect in polymerase activity and not to the defective RNP assembly process. Mini-vRNA template (NP-77) was expressed in HEK293T cells along with the polymerase constituted either with wildtype of mutant PB2 proteins and primer extension assay was performed in order to monitor vRNA (replication) and mRNA (transcription) synthesis in absence of NP. As evidenced

357 clearly, PB2 mutants shows various degrees of defects in vRNA synthesis (Fig.7A \& B), 358 which could perfectly be correlated with their ability to support polymerase activity 359 (evidenced in reporter assay, Fig 3.C) and RNP assembly process. Synthesis of mRNA was almost completely abrogated for all of the mutants (only AEA mutant showed minimal activity) (Fig7.A \& B), which could be a consequence of the low abundance of the vRNA 
defect in RNA synthesis activity of the polymerase harboring the PB2 mutant proteins with alteration in their SES motif.

\section{Introduction of human virus specific residues in the bat virus PB2 protein boosts} the activity of the chimeric polymerase

Extensive investigation of the molecular mechanism revealed that the highly conserved S-E-S motif, specifically the E282 residue, in the mid-link region of the PB2 is critical in supporting optimum activity of classical influenza A virus polymerase. Alteration of the SES motif via introduction of the bat specific serine residue at 282 position significantly restricts polymerase activity and hence inhibits virus propagation not only in human but also in bat cells (Fig3.C). Similar attenuation was also observed in case of viruses harboring the bat specific serine residue at the 627 position (Fig3.C)(21). Based upon these observations we hypothesize that the serine residues at critical positions of PB2 may serve as the key restriction elements present within the bat virus polymerase that are responsible for the lower replication fitness of bat influenza virus in comparison to the classical influenza A viruses.

To test our hypothesis, we reconstituted a chimeric polymerase composed of the PB1 and PA subunits of the $A / H 1 N 1 / W S N / 1933$ strain and the PB2 subunit of the A/H17N10/guatemala/060 strain. Influenza A/H1N1/WSN/1933 virus RNPs were reconstituted either with wild type or chimeric polymerases in HEK293T cells as described earlier (13). As evidenced, introduction of the bat virus PB2 in the human virus RNP severely restricts the polymerase resulting in around two logs decrease in reporter activity (Fig8.A). Although an increasing concentration of the H17N10 PB2 results in a dose 
dependent increase in reporter activity, the chimeric polymerase still remained attenuated with respect to the wild type, suggesting the severe restriction imposed by the bat PB2 upon the other subunit of the polymerase. This is in corroboration with the results presented by other groups, where alteration of the individual RNP component of human adapted influenza virus with that of the bat influenza virus restricted viral RNA synthesis (Pool et al, 2014; Juozapaitis et al., 2014; Zhou et al., 2014). Subsequently, we have replaced the bat specific serine

residues at 627 and 282 positions with human signature lysine and glutamic acid residues in the H17N10 PB2 protein in order to generate either single mutants (S627K and S282E) or double mutant (S672K/ S282E). Chimeric polymerases and RNPs were reconstituted either with wildtype or mutant H17N10 PB2 proteins to measure their ability to support viral RNA synthesis. Interestingly, substituting of the bat virus specific serine residues with human virus specific glutamic acid or lysine boosted the polymerase activity by 100 percent and 50 percent respectively thereby providing evidence that serine at 282 and 627 position of H17N10 PB2 protein may restrict the activity of bat influenza polymerases in human cells (Fig8.B). Generation of the double mutant S282E/S627K, harboring both of the human virus specific signature residues, showed minor enhancement compared to the single mutants, hence suggesting that these restriction factors in the polymerase does not act in cumulative fashion. Together, our data identified the PB2 S282 as a key residue which in association with PB2 S627 restricts the activity of the bat influenza PB2 protein and hence virus polymerase in human cells. 


\section{Discussion}

410

411

412

413

414

415

416

417

418

419

420

421

422

423

424

425

426

427

428

429

430

431

Higher genetic plasticity of the bat influenza viruses has been shown to facilitate their rapid adaptation in mice $(26,27)$, hence revealing their potential of cross species transmission in non-bat host species. Interestingly, these adapted bat viruses replicate poorly in ferrets $(26,27)$ and in human derived cell lines $(26)$, which could be attributed to the suboptimal activity of the viral polymerase in non-bat host species (7). In this work, we have identified a unique molecular signature in the PB2 subunit of bat influenza virus polymerase, which is responsible for its restricted activity in human cell lines. Extensive sequence and structural comparison between different influenza virus polymerases led us to the identification of a previously uncharacterized "S-E-S" motif in the PB2 subunit, which is completely conserved in conventional influenza A viruses, but is replaced with a "S-S-T" motif in bat influenza virus. Alteration of this canonical "S-E-S" motif with bat like "S-S-S" significantly reduced the polymerase activity and hence the fitness of influenza A/H1N1/WSN virus. In corroboration with these results, replacing the bat specific "S-S-T" with semi-canonical "S-E-T" motif results in significant enhancement in the activity of the chimeric polymerase reconstituted with PB1 and PA from conventional IAV and PB2 from bat IAV. Extensive investigation of the molecular mechanism revealed that the complete integrity of the S-E-S motif, specifically the negatively charged glutamic acid at the center of the motif, is critical for supporting RNA synthesis activity of the polymerase and replacing this glutamic acid either with alanine or with the bat specific serine residue severely attenuates the same. Clearly, this S-E-S motif, specifically the $282^{\text {nd }}$ E residues serves as a key molecular determinants of the fitness of classical influenza A virus polymerase in a host independent manner. On the other hand, our work also suggests 
432 that the serine at the $282^{\text {nd }}$ position of the bat influenza virus PB2 may serve as inherent 433 restriction element impeding its activity in human cells. It is interesting to note that the bat 434 specific serine residue at the PB2 $627^{\text {th }}$ position also imparts similar restriction to the viral polymerase activity in a host independent manner.

436 The evolutionary history of the bat influenza viruses and their ancestry relationship with 437 other flu variants are not clear (16). However, the new world bat influenza viruses 438 (H17N10 and H18N11 subtypes) have been designated as distinctly divergent entity of 439 IAVs $(7,8)$. As per the phylogenetic backdating, the new world bat IAVs possibly have separated from all of the conventional lineages around 650 years ago (16). This is

441 supported by the fact that the amino acid sequences of the internal segments of the new 442 world bat viruses tolerate higher diversity in contrast to the conventional IAVs and hence 443 form a distinct outgroup (8). It is possible that the bat influenza viruses served as the 444 ancestor for the all other IAVs, which during the course of adaptation into other host 445 species acquired species specific signatures in different internal gene segments. The 446 change of serine to glutamic acid at $627^{\text {th }}$ (avian) and $282^{\text {nd }}$ positions of PB2 may thus 447 had served as crucial adaptation signatures of the virus from bat to not-bat hosts, which 448 have conferred additional fitness to the viral RNA polymerase in terms of replication in 449 corresponding host species. In another possible scenario, bat and non-bat IAVs may have 450 segregated from a common ancestor (27), where the bat specific serine residues may got 451 selected over glutamic acid in order to generate a slower replication variant of the 452 influenza virus, which might be suitable in terms of establishing host-parasite equilibrium 453 in the bat species. It is interesting that the old-world bat IAVs (H9N2), which shows 454 relatively lesser divergence from the conventional variants (16), harbors glutamic acid at 
455 the $627^{\text {th }}$ and $282^{\text {nd }}$ positions of PB2 (28), further supporting the importance of these 456 residues in adaptation of the viruses from bat to non-bat hosts or vice versa.

457 It is interesting to note that bat influenza virus polymerase, specifically the PB2 subunit, 458 harbors higher number of serine residues in the solvent exposed surface in comparison 459 to the conventional IAVs (table 1,). For example, all of the aspartic acid or glutamic acid 460 residues identified in this study are serine or threonine in bat virus polymerase. It is 461 tempting to speculate that phosphorylation of some of these serine residues, specifically 462 at the $627^{\text {th }}$ and $282^{\text {nd }}$ positions, is important for proper functionality of the polymerase 463 and that is why these residues may get adapted to negatively charged glutamic acid or 464 aspartic acid residues in conventional IAVs, so that the dependence over the host kinases 465 could be bypassed. In opposite sense, a glutamic acid to serine adaptation could also 466 impart functional regulation to the bat virus polymerase, which could be controlled via 467 phosphorylation by host kinases. A comparative phospho-proteome analyses of the of 468 the bat and non-bat influenza virus polymerase in various host species may shade light 469 upon this possibility of such naturally occurring phosphomimetic adaptation sites in the 470 conventional influenza A viruses. From a different perspective, mutation of these serine 471 residues to glutamic acid within the bat influenza virus polymerase may also increase its 472 replication fitness, which together with the adaptive mutations in the HA and NA $(26,27)$ 473 could lead to the spillover of the bat viruses in non-bat host species. 
477 Human embryonic kidney (HEK293T), Madin-Darby Canine Kindey (MDCK), Human lung

478 epithelial carcinoma(A549), Pteropus alecto kidney (PaKi) cells were maintained in

479 DMEM (Gibco), supplemented with 10\% FBS, 1X Penicillin -Streptomycin solution and $4801 \mathrm{X}$ GlutaMAX at $37^{\circ} \mathrm{C}$ temperature, $98 \%$ relative humidity, in presence of $5 \% \mathrm{CO}$. DF4811 (chicken fibroblast) cells were maintained at $39^{\circ} \mathrm{C}$ temperature, $98 \%$ relative humidity, 482 in the same culture media.

\section{Plasmids and viruses:}

484 All genes were derived from the influenza A (A/WSN/33) or influenza B 485 (B/Brisbane/60/2008) viruses. Polymerase proteins and NP were expressed in cells from 486 the plasmids, pCDNA3-PB2-FLAG (encoding a C-terminal FLAG tag), pCDNA3-PA, 487 pCDNA3-PB1, pCDNA6.2-NP-V5 (encoding C terminal V5 tag for Influnza A) and 488 pcDNA3.1-NP for Influenza B. The PB2 ORF of Influenza A/H17N10/guatemala/060 was 489 cloned also under the CMV promotor in pCDNA3.1 vector with $\mathrm{C}$ terminal $3 \mathrm{X}$ FLAG 490 epitope tag. vNA-luc reporter plasmids encodes firefly luciferase in the negative sense 491 flanked by UTRs from the NA gene derived from $A / H 1 N 1 / W S N / 1933$ and 492 B/Brisbane/60/2008 cloned under RNA Polymerase I promoter and terminator in pHH21 493 vector system for expression in HEK293T cells and in pGHH21 for expression in DF1 494 cells. Viruses were prepared using the pBD bi-directional reverse genetics system using 495 pBD plamids for polymerase subunits and NP and pTM $\Delta$ RNP for expression of all other 496 segments.

\section{Site directed mutagenesis:}


498 Site directed mutagenesis primers were designed by quick change primer design 499 software (Agilent technologies). Mutation was performed by PCR amplification of the 500 plasmids using Pfu Turbo DNA Polymerase enzyme (Agilent technologies) followed by

501 Dpnl digestion and transformation in E. coli DH5a cells. Mutations were confirmed by 502 Sanger sequencing.

\section{Polymerase Activity Assays:}

504 HEK293T or DF-1 cells were reverse transfected in triplicates using Lipofectamine 3000 505 with plasmids expressing PA, PB1, PB2-FLAG,NP-v5/NP and vNA-Luc. Cells were 506 harvested 36 h.p.t, polymerase activity measured using the Luciferase Assay System 507 (Promega) on GloMax20/20 luminometer (Promega). Equivalent PB2 and NP protein 508 expression were confirmed by western blotting.

Rescue of recombinant viruses and plaque assay:

510 Co-cultures of HEK293T and MDBK cells were reverse transfected using 511 Lipofectamine3000 Reagent with virus rescue plasmids pTM $\Delta R N P, p B D \_P B 2-F L A G 143$, 512 pBD*_PB1, pBD_PA and pBD_NP. 24 h.p.t, the culture media was replaced with Virus 513 Growth Media (1X DMEM, 1X PenStrep, 4 mM GlutaMAX, 0.2\% Bovine Serum Albumin 514 (BSA), 25 mM HEPES buffer and $0.5 \mu \mathrm{g} / \mathrm{ml}$ TPCK Trypsin). Supernatants were harvested 515 48-72 h.p.t and the viruses subsequently amplified in MDBK cells. Plaque assay was 516 performed in MDCK cells. 0.3million cells were seeded in each well of 12 well cell culture 517 plate day before the assay. Virus stocks were diluted in 10-fold series in virus growth 518 media. Cells were infected by the dilutions for 50 minutes at $37^{\circ} \mathrm{C}$ with intermittent shaking 519 in each 10 minutes. After 50 minutes the infected cells were overlaid with 1:1 mixture of 
2.4\% avicel and 2X DMEM (2X DMEM, 1X PenStrep, 4 mM GlutaMAX, 0.4\% Bovine Serum Albumin (BSA), 50 mM HEPES buffer and $1.0 \mu \mathrm{g} / \mathrm{ml}$ TPCK Trypsin). Plates were harvested at 72 hours post infection, fixed with $70 \%$ ethanol and stained with $1 \%$ crystal violet. Plaques were counted from the stained plates and titer was calculated.

\section{Multi cycle replication kinetics:}

A549, MDCK and PAKI cell lines were infected with wild type or mutant Influenza $\mathrm{A} / \mathrm{H} 1 \mathrm{~N} 1 / \mathrm{WSN} / 1933$ viruses at $\mathrm{MOI}$ of 0.01 in three biological replicates. Supernatants were harvested at $8,16,24,48$ and 72 hours post infection. Viral titer in the supernatant was quantitated by performing plaque assay in MDCK cells.

\section{RNA Isolation, Reverse Transcription and PCR:}

Supernatants from virus rescue experiments were harvested, clarified by centrifugation at 12,000 $\mathrm{g}$ for 10 minutes, and viral RNA was extracted using TRIzol reagent (Invitrogen). Reverse transcription was performed by M-MLV RT PB2-specific primers. PCR amplification of cDNA was done by Phusion DNA Polymerase with PB2-gene specific primer pair. PCR amplified fragments were used for sanger sequencing.

Primer Extension: PB1,PA and wild type or mutant PB2 encoding plasmids with a small 77nucletide long viral RNA with 3' and 5' UTR of NP (NP77) expression plasmid were transfected in HEK293T cells. 48 hours post transfection the cells were harvested. Total RNA was isolated by TRIzol reagent (Invitrogen). Primer extension was performed by Superscript III reverse transcriptase enzyme (Thermo) and fluorescence labelled primers as described earlier(25). The reactions were separated in Urea-PAGE and the gel imaged in Bio-Rad chemidoc. 


\section{Co-immunoprecipitations:}

543 HEK293T cells were transfected for RNP reconstitution with expression plasmids 544 encoding NP-v5, PB1, PA and FLAG-tagged WT or mutant PB2, along with vRNA (NA 545 segment). Cells were lysed 48 h.p.t. using Co-IP buffer (50 mM Tris- $\mathrm{HCl}[\mathrm{pH} 7.4], 150$ $546 \mathrm{mM} \mathrm{NaCl}, 1 \mathrm{mM}$ EDTA, 1\% NP-40, 1\% Na-deoxycholate, 0.1\% SDS) supplemented with $5471 \mathrm{X}$ protease inhibitor cocktail $(10 \mu \mathrm{L}$ of $50 \mathrm{X} \mathrm{PI}$ in $500 \mu \mathrm{L}$ of Co-IP buffer), Halt 548 phosphatase inhibitor $\left(10 \mu \mathrm{L}\right.$ for $500 \mathrm{uL}$ of lysis buffer), and incubated at $4{ }^{\circ} \mathrm{C}$ for 20 549 minutes on a rocker. Lysates were clarified by centrifuging at $20,000 \mathrm{~g}$ for 20 minutes at $4^{\circ} \mathrm{C}$. Total protein samples were separated from the lysate. Lysates were supplemented

551 with $0.5 \mathrm{mg} / \mathrm{mL}$ BSA and precleared with $20 \mathrm{uL}$ of pre-equilibrated Protein $\mathrm{A}$ agarose 552 beads. After preclearing the lysates were incubated overnight with the antibody. Next day, 553 pre-equilibrated protein A magnetic beads were added to the lysate and incubated for 1 554 hour. Protein-A beads were then recovered using magnetic racks and washed thrice with 555 Co-IP buffer. Finally, sample beads were treated with $30 \mu \mathrm{L}$ of $5 X$ Laemmli buffer, heated 556 at $98^{\circ} \mathrm{C}$ for 5 minutes, centrifuged at $10,000 \mathrm{~g}$ for 10 minutes. Samples were separated 557 by SDS-PAGE and identified by Western blotting.

\section{Western blotting:}

560 Cell lysates were separated by SDS-PAGE and transferred to methanol-activated PVDF 561 membrane (Bio-Rad) using Trans-Blot Turbo Transfer System. The membrane was 562 blocked in 5\% skimmed milk solution in 1X TBST at room temperature for 2 hours. 563 Primary antibody incubation done at $4{ }^{\circ} \mathrm{C}$ in rocking condition for overnight. Next day, after 564 washing three times with 1X TBST, the membrane was incubated with HRP-conjugated 
565

566

567 incubated for 3-5 minutes and developed in BioRad chemi-doc.

secondary antibody at room temperature for 1 hour. Before developing, the blots were washed thrice with $1 \mathrm{X}$ TBST. Chemiluminescent substrate applied over the blot and

\section{Immunofluorescence Assay:}

569

570

571

572

573

574

575

576

577

578

579

580

581

582

583

584

585

586

A549 cells grown on coverslips were transfected with plasmids encoding wild type or mutant PB2 proteins. 24 hours post-transfection, cells were washed in PBS, fixed with $3 \%$ formaldehyde (20 minutes at room temperature), quenched with $0.1 \mathrm{M}$ Glycine, permeabilized with $0.1 \%$ Triton-X 100 in PBS (10 minutes at room temperature). After blocking with $3 \%$ BSA (20 minutes at RT), cells were incubated with primary antibody (anti-FLAG) for 1 hour at room temperature and washed thrice. Secondary antibody (Alexa Fluor 488-conjugated donkey anti-mouse $\operatorname{lgG}$ ) incubation done for 40 minutes at room temperature. DAPI staining was done along with the secondary antibody incubation. After secondary antibody incubation cells were washed thrice with PBS and once with nano-pure water and mounted on slide with Fluoroshield (Sigma Aldrich). Images were taken in fluorescence microscope (Leica).

\section{Bioinformatics and structural analysis:}

Specific PB2 protein sequences were obtained from Influenza Research database (www.fludb.org) by selecting data type-protein, Virus type-A, proteins-PB2 and separately selecting Host-Human( $n=36243)$, Avian( $n=19762)$ and Bat(n=7). The aligned FASTA files were viewed and analyzed in Bioedit sequence alignment editor(29). Multiple sequence alignment was performed in Bioedit software by using ClustalW followed by manual trimming. Logo plots were generated by WebLogo server (weblogo.berkeley.edu/logo.cgi 
587 )using the aligned FASTAfiles (http://www.ebi.ac.uk/pdbe/prot int/pistart.html) (30).

588 PDBePISA tool (31) was used to analyze the solvent assessible surface area of PB2

589 proteins from the following PBD file- 4WSB,4WSA,6RR7 and 5D98(19, 20, 32). Structural

590 alignment of the PB2 protein from trimeric polymerase crystal structure was performed in

591 UCSF Chimera software.

592 Statistical analysis and data analysis:

593 Graph preparations and statistical analysis were done in Microsoft Excel software. 594 Densitometric analysis was performed in Bio-Rad Image Lab software.

\section{Acknowledgement}

597 We sincerely acknowledge Prof. Andrew Mehle (University of Wisconsin Madison) for his 598 comments on the manuscript and providing valuable resources. A.M. thanks DBT, 599 Ramalingaswami re-entry fellowship (BT/RLF/Re-entry/02/2015), SERB, Early Career 600 Research Award (ECR/2017/001896) and MHRD, "Scheme for Transformational and 601 Advanced Research in Science" \{STARS/APR2019/BS/369/FS (Project ID: 369)\} for 602 financial support. Individual fellowship for SB (File No.09/081(1301)/2017-EMR603 I_),NK(File No.09/081(1316)/2017-EMR-I_) and AD(File No.09/081(1405)/2020-EMR-I_) was provided by Council of Industrial and Fundamental research, Govt. of India. We 605 sincerely acknowledge Dr. Gayatri Mukherjee from School of Medical science and 606 Technology, IIT Kharagpur, for providing fluorescence based gel imaging facility. 
609

610

611

612

613

614

615

616

617

618

619

620

621

622

623

624

625

626

627

628

629

\section{Reference}

1. Long JS, Mistry B, Haslam SM, Barclay WS. 2019. Host and viral determinants of influenza A virus species specificity. Nat Rev Microbiol.

2. Rogers GN, Paulson JC. 1983. Receptor determinants of human and animal influenza virus isolates: Differences in receptor specificity of the $\mathrm{H} 3$ hemagglutinin based on species of origin. Virology 127:361-373.

3. Cauldwell A V., Long JS, Moncorgé O, Barclay WS. 2014. Viral determinants of influenza A virus host range. J Gen Virol 95:1193-1210.

4. Subbarao EK, London W, Murphy BR. 1993. A single amino acid in the PB2 gene of influenza A virus is a determinant of host range. J Virol 67:1761-1764.

5. Almond JW. 1977. A single gene determines the host range of influenza virus. Nature 270:617-618.

6. Mehle A, Doudna JA. 2009. Adaptive strategies of the influenza virus polymerase for replication in humans. Proc Natl Acad Sci U S A 106:21312-21316.

7. Tong S, Li Y, Rivailler P, Conrardy C, Alvarez Castillo DA, Chen LM, Recuenco S, Ellison JA, Davis CT, York IA, Turmelle AS, Moran D, Rogers S, Shi M, Tao Y, Weil MR, Tang K, Rowe LA, Sammons S, Xu X, Frace M, Lindblade KA, Cox NJ, Anderson LJ, Rupprecht CE, Donis RO. 2012. A distinct lineage of influenza A virus from bats. Proc Natl Acad Sci U S A 109:4269-4274.

8. Tong S, Zhu X, Li Y, Shi M, Zhang J, Bourgeois M, Yang H, Chen X, Recuenco S, Gomez J, Chen LM, Johnson A, Tao Y, Dreyfus C, Yu W, McBride R, Carney PJ, 
Gilbert AT, Chang J, Guo Z, Davis CT, Paulson JC, Stevens J, Rupprecht CE, Holmes EC, Wilson IA, Donis RO. 2013. New World Bats Harbor Diverse Influenza A Viruses. PLoS Pathog 9.

9. Sun X, Shi Y, Lu X, He J, Gao F, Yan J, Qi J, Gao GF. 2013. Bat-derived Influenza Hemagglutinin H17 Does Not Bind Canonical Avian or Human Receptors and Most Likely uses a Unique Entry Mechanism. CellReports 3:769778.

10. García-Sastre A. 2012. The neuraminidase of bat influenza viruses is not a neuraminidase. Proc Natl Acad Sci 109:18635-18636.

11. Karakus U, Thamamongood T, Ciminski K, Ran W, Günther SC, Pohl MO, Eletto D, Jeney C, Hoffmann D, Reiche S, Schinköthe J, Ulrich R, Wiener J, Hayes MGB, Chang MW, Hunziker A, Yángüez E, Aydillo T, Krammer F, Oderbolz J, Meier M, Oxenius A, Halenius A, Zimmer G, Benner C, Hale BG, García-Sastre A, Beer M, Schwemmle M, Stertz S. 2019. MHC class II proteins mediate crossspecies entry of bat influenza viruses. Nature 567:109-112.

12. Giotis ES, Carnell G, Young EF, Ghanny S, Soteropoulos P, Wang LF, Barclay WS, Skinner MA, Temperton N. 2019. Entry of the bat influenza H17N10 virus into mammalian cells is enabled by the MHC class II HLA-DR receptor. Nat Microbiol 4:2035-2038.

13. Poole DS, Yu S, Cai Y, Dinis JM, Muller MA, Jordan I, Friedrich TC, Kuhn JH, Mehle A. 2014. Influenza A Virus Polymerase Is a Site for Adaptive Changes during Experimental Evolution in Bat Cells. J Virol 88:12572-12585. 
652

653

654

655

656

657

658

659

660

661

662

663

664

665

666

667

668

669

670

671

672

673

14. Zhou B, Ma J, Liu Q, Bawa B, Wang W, Shabman RS, Duff M, Lee J, Lang Y, Cao N, Nagy A, Lin X, Stockwell TB, Richt JA, Wentworth DE, Ma W. 2014.

Characterization of Uncultivable Bat Influenza Virus Using a Replicative Synthetic Virus. PLoS Pathog 10.

15. Juozapaitis M, Aguiar Moreira É, Mena I, Giese S, Riegger D, Pohlmann A, Höper D, Zimmer G, Beer M, García-Sastre A, Schwemmle M. 2014. An infectious batderived chimeric influenza virus harbouring the entry machinery of an influenza A virus. Nat Commun 5.

16. Ciminski K, Pfaff F, Beer M, Schwemmle M. 2020. Bats reveal the true power of influenza A virus adaptability. PLoS Pathog 16:1-7.

17. Lutz A, Dyall J, Olivo PD, Pekosz A. 2005. Virus-inducible reporter genes as a tool for detecting and quantifying influenza A virus replication. J Virol Methods 126:13-20.

18. Kirui J, Bucci MD, Poole DS, Mehle A. 2014. Conserved Features of the PB2 627 Domain Impact Influenza Virus Polymerase Function and Replication. J Virol 88:5977-5986.

19. Fan H, Walker AP, Carrique L, Keown JR, Serna Martin I, Karia D, Sharps J, Hengrung N, Pardon E, Steyaert J, Grimes JM, Fodor E. 2019. Structures of influenza A virus RNA polymerase offer insight into viral genome replication. Nature 573:287-290.

20. Pflug A, Guilligay D, Reich S, Cusack S. 2014. Structure of influenza A polymerase bound to the viral RNA promoter. Nature 
https://doi.org/10.1038/nature14008.

21. Chin AWH, Li OTW, Mok CKP, Ng MKW, Peiris M, Poon LLM. 2014. Influenza A viruses with different amino acid residues at PB2-627 display distinct replication properties in vitro and in vivo: Revealing the sequence plasticity of PB2-627 position. Virology 468:545-555.

22. Dlugolenski D, Jones L, Tompkins SM, Crameri G, Wang LF, Tripp RA. 2013. Bat cells from Pteropus alecto are susceptible to influenza A virus infection and reassortment. Influenza Other Respi Viruses 7:900-903.

23. Deng T, Engelhardt OG, Thomas B, Akoulitchev A V., Brownlee GG, Fodor E. 2006. Role of Ran Binding Protein 5 in Nuclear Import and Assembly of the Influenza Virus RNA Polymerase Complex. J Virol 80:11911-11919.

24. Tarendeau F, Boudet J, Guilligay D, Mas PJ, Bougault CM, Boulo S, Baudin F, Ruigrok RWH, Daigle N, Ellenberg J, Cusack S, Simorre JP, Hart DJ. 2007. Structure and nuclear import function of the C-terminal domain of influenza virus polymerase PB2 subunit. Nat Struct Mol Biol 14:229-233.

25. Turrell L, Lyall JW, Tiley LS, Fodor E, Vreede FT. 2013. The role and assembly mechanism of nucleoprotein in influenza A virus ribonucleoprotein complexes. Nat Commun 4:1-11.

26. Zhong G, Fan S, Hatta M, Nakatsu S, Walters KB, Lopes TJS, Wang JI-H, Ozawa M, Karasin A, Li Y, Tong S, Donis RO, Neumann G, Kawaoka Y. 2019. Mutations in the Neuraminidase-Like Protein of Bat Influenza H18N11 Virus Enhance Virus Replication in Mammalian Cells, Mice, and Ferrets. J Virol 94:1-12. 
696

697

698

699

700

701

702

703

704

705

706

707

708

709

710

711

712

713

714

715

27. Ciminski K, Ran W, Gorka M, Lee J, Malmlov A, Schinköthe J, Eckley M, Murrieta RA, Aboellail TA, Campbell CL, Ebel GD, Ma J, Pohlmann A, Franzke K, Ulrich R, Hoffmann D, García-Sastre A, Ma W, Schountz T, Beer M, Schwemmle M. 2019. Bat influenza viruses transmit among bats but are poorly adapted to non-bat species. Nat Microbiol 4:2298-2309.

28. Kandeil A, Gomaa MR, Shehata MM, El Taweel AN, Mahmoud SH, Bagato O, Moatasim Y, Kutkat O, Kayed AS, Dawson P, Qiu X, Bahl J, Webby RJ, Karesh WB, Kayali G, Ali MA. 2018. Isolation and Characterization of a Distinct Influenza A Virus from Egyptian Bats. J Virol 93:1-10.

29. Thomas A. Hall. 1999. BioEdit: a user-friendly biological sequence alignment editor and analysis program for Windows 95/98/NT. Nucleic Acids Symp Ser No 41.

30. Crooks GE, Hon G, Chandonia JM, Brenner SE. 2004. WebLogo: A sequence logo generator. Genome Res 14:1188-1190.

31. Krissinel E, Henrick K. 2007. Protein interfaces, surfaces and assemblies' service PISA at the European Bioinformatics Institute. J Mol Biol 372:774-797.

32. Hengrung N, El Omari K, Serna Martin I, Vreede FT, Cusack S, Rambo RP, Vonrhein C, Bricogne G, Stuart DI, Grimes JM, Fodor E. 2015. Crystal structure of the RNA-dependent RNA polymerase from influenza C virus. Nature 527:114117. 


\section{Figure legends and tables}

Figure 1. Bat specific serine at PB2-627 position restricts the polymerase in human but not in avian cells. A. Influenza A virus RNPs are the major determinant of its host specific replication fitness. The $627^{\text {th }}$ amino acid of the PB2 subunit of polymerase harbors a lysine $(\mathrm{K})$ or a glutamic acid $(\mathrm{E})$ residues for human or avian adapted viruses respectively. Bat influenza viruses harbors a serine (S) at the same position. B. Luciferase based reporter assay was performed to assess the polymerase function in human and chicken cells. Viral RNP, with the genetic background of A/WSN/1933, was reconstituted in HEK293T or DF1 cells either with wildtype PB2 containing a lysine, or mutant PB2 containing glutamic acid or serine residue at 627 th position. $n=3 \pm$ standard deviation. ${ }^{*} p<0.05$ one-way ANOVA when compared to PB2-627K.

Table1: Analysis of surface exposed serine and threonine residues in H17N10 and H3N2 PB2 from the crystal structure of RNA dependent RNA polymerase (4WSB.PDB \& 6RR7.PDB).

Criteria

Number
Position 


\begin{tabular}{|c|c|c|}
\hline $\begin{array}{l}\text { Total number of } \\
\text { surface exposed } \\
\text { serine and } \\
\text { threonine } \\
\text { residues in } \\
\text { H17N10-PB2 } \\
\text { (4WSB.PDB) }\end{array}$ & $\begin{array}{l}\text { Serine (34), } \\
\text { Threonine (34) }\end{array}$ & $\begin{array}{l}\text { Serine- } \\
\text { 14,61,92,156,178,179,225,226,279,282,288, } \\
290,291,300,322,324,366,371,442,443,481,5 \\
14,533,534,544,593,622,674,678,683,684,68 \\
5,688,741 \\
\text { Threonine- } \\
76,79,129,155,184,186,224,227,235,245,286 \\
\text { 287,303,340,346,351,353,355,390,400,444, } \\
451,468,472,549,562,566,569,582,596,598,6 \\
09,631,681\end{array}$ \\
\hline $\begin{array}{l}\text { Total number of } \\
\text { surface exposed } \\
\text { serine and } \\
\text { threonine } \\
\text { residues in } \\
\text { H3N2-PB2 } \\
\text { (6RR7.PDB) }\end{array}$ & $\begin{array}{l}\text { Serine (20) } \\
\text { Threonine (20) }\end{array}$ & $\begin{array}{l}\text { Serine - } \\
14,92,179,225,226,279,286,322,324,481,514 \\
, 533,534,544,593,684,688,79,155,286,582 \\
\text { Threonine- } \\
\text { 178,371,674,683,76,129,184,186,224,235,24 } \\
5,287,303,346,351,468,549,569,598,609\end{array}$ \\
\hline
\end{tabular}




\begin{tabular}{|l|l|l|}
\hline Surface exposed & Serine (17) & Serine- \\
Serine and & Threonine (18) & $61,156,178,282,288,290,291,300,366,371,44$ \\
Threonine & $2,443,622,674,678,683,685$ \\
residue in & Threonine- \\
H17N10-PB2 & $76,155,227,286,340,353,355,390,400,444,45$ \\
changed to other & $1,472,562,566,582,596,631,681$ \\
residue in & & \\
classical & & \\
influenza virus & & \\
PB2 & Aspartic acid & Glutamic acid- \\
\hline $\begin{array}{l}\text { Surface exposed } \\
\text { Serine and }\end{array}$ & $(2)$ & Glutamic acid \\
Threonine & Aspartic acid- \\
residue of & (3) & \\
H17N10-PB2 & & \\
changed to & & \\
aspartic and & & \\
glutamic residue & & \\
in classical & & \\
PB2 & & \\
\hline
\end{tabular}


733

734

735

736

737

738

739

740

741

742

743

744

745

746

747

748

749

750

751

752

753

754

755

\section{Figure 2. Identification of species-specific signature residues in PB2 subunit of bat} influenza virus polymerase. A. Residue wise solvent accessible surface area (SASA in $\AA^{2}$ ) of $\mathrm{H} 17 \mathrm{~N} 10$ monomeric PB2 and in trimeric polymerase was calculated. Serine and threonine residues were plotted as blue dots on the basis of their exposed surface area in monomeric and trimeric form. Serine or threonine residues in bat virus PB2 protein, which are a glutamic acid or aspartic acid residue in conventional Influenza viruses are marked as red and yellow dots respectively. Logo-plots of the shortlisted amino acid residues (marked with Asterix) which represent highly conserved glutamic /aspartic acid in the PB2 protein of influenza A viruses of human (sequences analyzed: 36243 ) or avian (sequences analyzed: 19762) origin but harbors serine in the PB2 of bat viruses (sequences analyzed: 7). B. Spatial organization of the surface exposed glutamic laspartic acid residues in the heterotrimeric polymerase structure of a human adapted influenza $A$ virus (A/NT/60/1968,PDB ID:6RR7) that are shortlisted for functional screening. C Luciferase based reporter assay was performed to assess the polymerase function of the recombinant RNA dependent RNA polymerase with wild type or alanine substitution mutants of PB2 proteins using the genetic background of A/WSN/1933 strain. $n=3 \pm$ standard deviation. ${ }^{*} p<0.05$ one-way ANOVA when compared to wildtype PB2.

Figure 3. Conventional influenza A viruses, harboring bat specific signature serine residues are defective in bat and not-bat host species. Recombinant influenza A/H1N1/WSN/1933 was generated contains a serine or alanine residue at $282^{\text {nd }}$ position and serine at $627^{\text {th }}$ position of PB2. A. Plaque morphology of recombinant viruses contain the mutation. B. Madin Darby canine kidney, MDCK, C. Human lung epithelial carcinoma, A549 and D. Pteropus Alecto kidney, PaKi cells were infected with recombinant viruses 
756 at an MOI of 0.01 in three biological replicates. Supernatants were collected at 8,16,24,48

757 and 72 hours post infection. Viral titer was calculated for each time points by performing 758 plaque assay.

Figure 4. A surface exposed, highly conserved "S-E-S" motif is crucial for the activity of viral RNA polymerase. A. PB2 $282^{\text {nd }}$ residue is present in the a15 helix (highlighted in red color in the surface representation of the polymerase) of the mid-link region, which is completely surface exposed and structurally conserved in influenza $A$ 763 (red), bat infecting influenza (blue), influenza B (yellow) and influenza C (green) viruses.

764 The amino acid residues denoted are of influenza A. B. Multiple sequence alignment of 765 Influenza A/H17N10(A/H17N10/Guetamala/060), Influenza $\quad A \quad$ /H1N1 766 (A/H1N1/WSN/1933), influenza C (C/Aichi/1/81), influenza D (D/Quebec/1M-H/2019) and 767 influenza B (B/Brisbane/163/2008) showing the variable degrees of conservation of the S-E-S motif. C. Luciferase based reporter assay was performed to assess the RNA dependent RNA polymerase function (using the genetic background of influenza A/H1N1/WSN/1933 strain) in human and chicken cells reconstituted by wild type or

771 mutant PB2 protein. $n=3 \pm$ standard deviation. * $p<0.05$ one way ANOVA when compared

772 to wildtype PB2. D. Luciferase based reporter assay was performed to assess the 773 polymerase function of Influenza B/Brisbane/2008 RNA dependent RNA polymerase 774 containing wild type or mutant PB2 protein. $n=3 \pm$ standard deviation. ${ }^{*}<0.05$ one way 775 ANOVA when compared to wildtype PB2.

776 Figure 5. Alteration of the "SES" motif affects RNP reconstitution without 777 impacting interaction with other viral proteins, NP and PB1. A. RNP reconstitution 778 was performed by transfecting NP, PB1, PA, PB2 protein and vRNA expression plasmid 
779 in HEK293T cells. 48 hours post transfection cells were lysed and PB2 protein was

780

781

782

783

784

785

786

787

788

789

790

791

792

793

794

795

796

797

798

799

800

801

immunoprecipitated and blotted for co-precipitated NP. B.

B. PB2 and NP interaction was

checked by transfecting NP expressing plasmid along with wild type or mutant PB2 expressing plasmids in HEK293T cells. 48 hours post transfection cells were lysed and PB2 was immunoprecipitated and blotted for co-precipitated NP. C. PB2 and PB1 interaction was checked by transfecting PB1 expressing plasmid along with wild type or mutant PB2 expressing plasmids in HEK293T cells. 48 hours post transfection cells were lysed and PB2 was immunoprecipitated and blotted for co-precipitated PB1

Figure 6. Motif mutants shows similar subcellular localization to the wild type PB2

protein. Plasmids expressing wild type of mutant PB2 proteins were transfected in A549 cells. 24 hours post transfection cells were fixed and parmealized. Cells were first stained with anti-PB2 mouse antibody followed by Alexa fluore 488 anti-mouse rabbit antibody. Nucleus were stained with DAPI. Image were taken in Leica Fluorescence microscope.

Figure 7. The "SES" motif is critical for supporting RNA synthesis activity of the polymerase. A. primer extension was performed with a 77 nucleotide long mini viral RNA template containing 3' and 5' UTR of NP gene. Trimeric polymerase was reconstituted in HEK293T cells with wild type or Mutant PB2. Total RNA was extracted by TRIzol method and primer extension performed with fluorescently labelled specific primer for vRNA, mRNA and 5srRNA. Reaction products were analyzed by Urea-PAGE and imaged in BioRad chemidoc. B. Densitometric analysis of primer extension experiment from three independent experiments.

Figure 8. Introduction of S627K and S282E mutations in H17N10 PB2 protein boosts activity of the chimeric polymerase. A. Luciferase based reporter assay was 
802 performed by reconstituting the Influenza A/H1N1/WSN/1933 RNP in HEK293T, either 803 with H1N1 PB2 or with different amount of H17N10 PB2 expression plasmid. $804 n=3 \pm$ standard deviation. ${ }^{*} p<0.05$ one way ANOVA when compared to previous set. B. 805 Luciferase based reporter assay was performed by reconstituting the Influenza 806 A/H1N1/WSN/1933 RNP with either wild type H17N10 PB2 or mutant H17N10 PB2 807 protein. $n=3 \pm$ standard deviation. ${ }^{*} \mathrm{p}<0.05$ one-way ANOVA when compared to PB2 808 wildtype or S282E or S627K. 
A

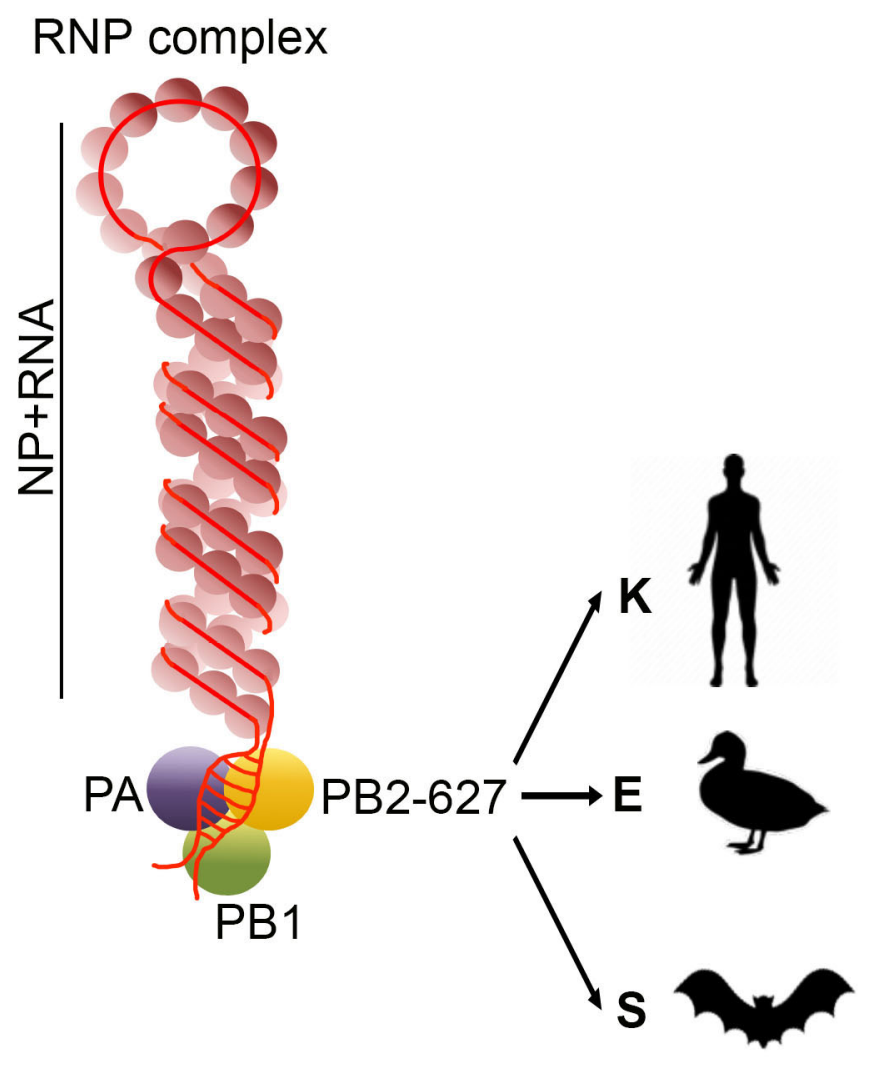

B
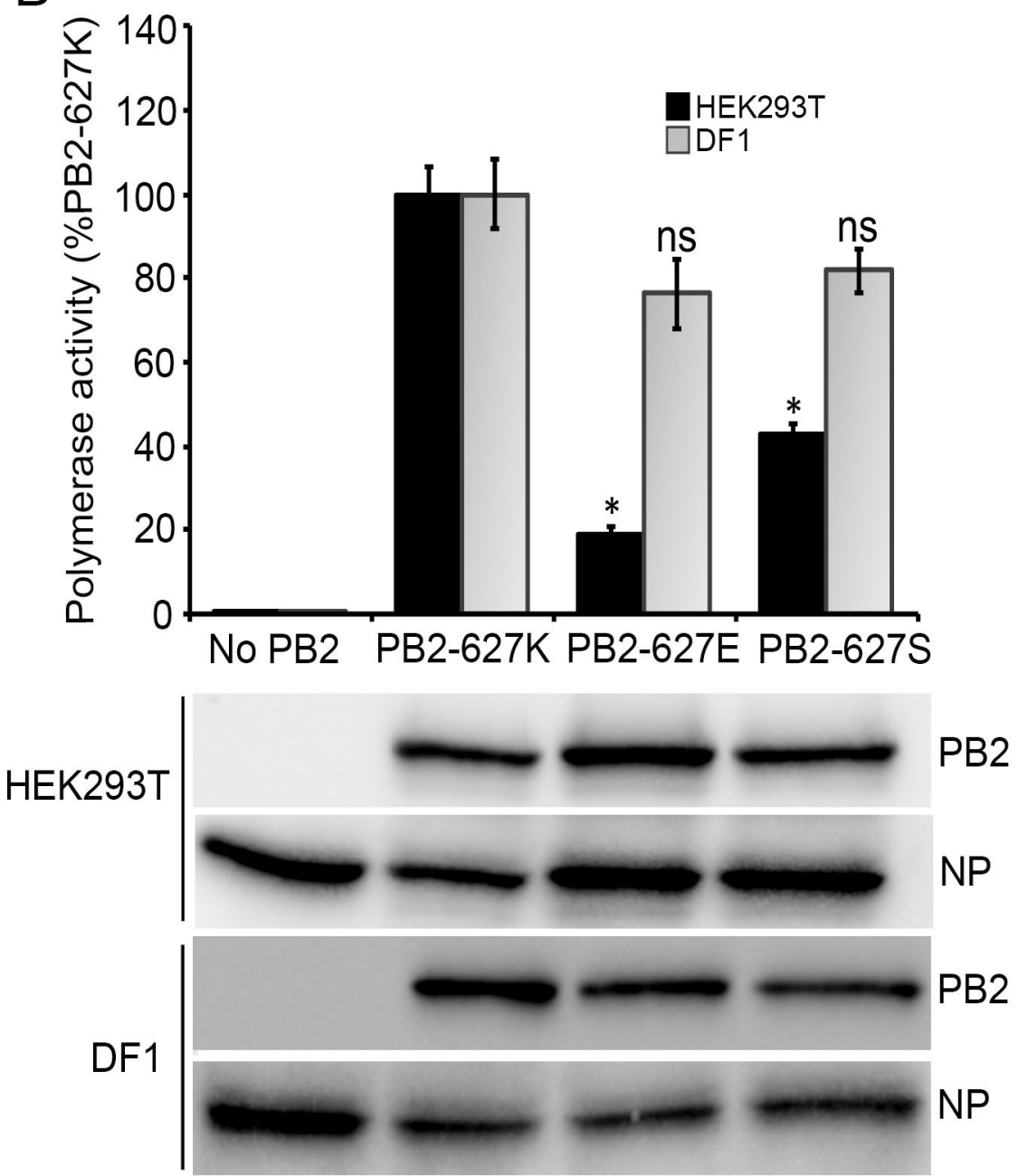


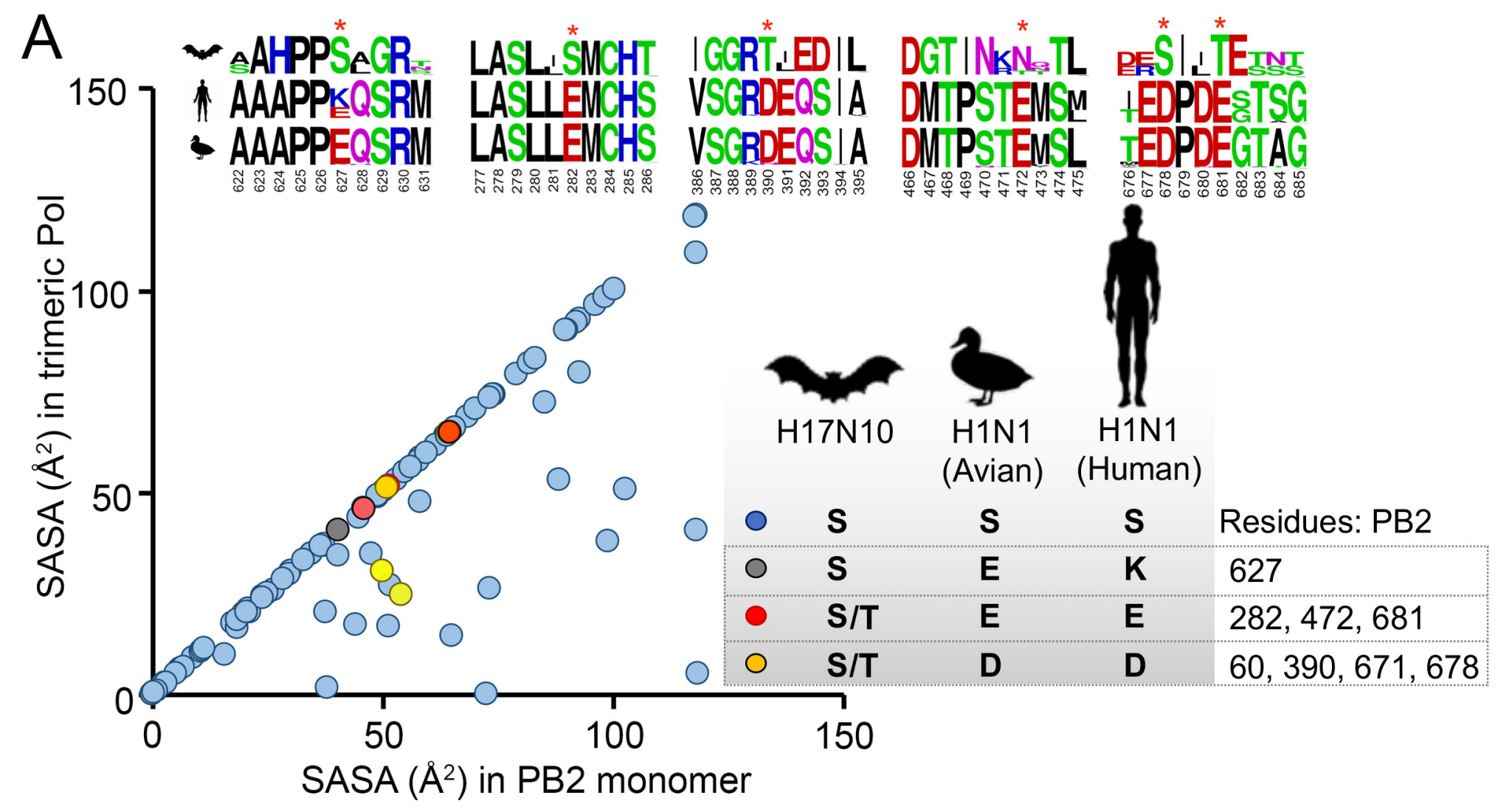

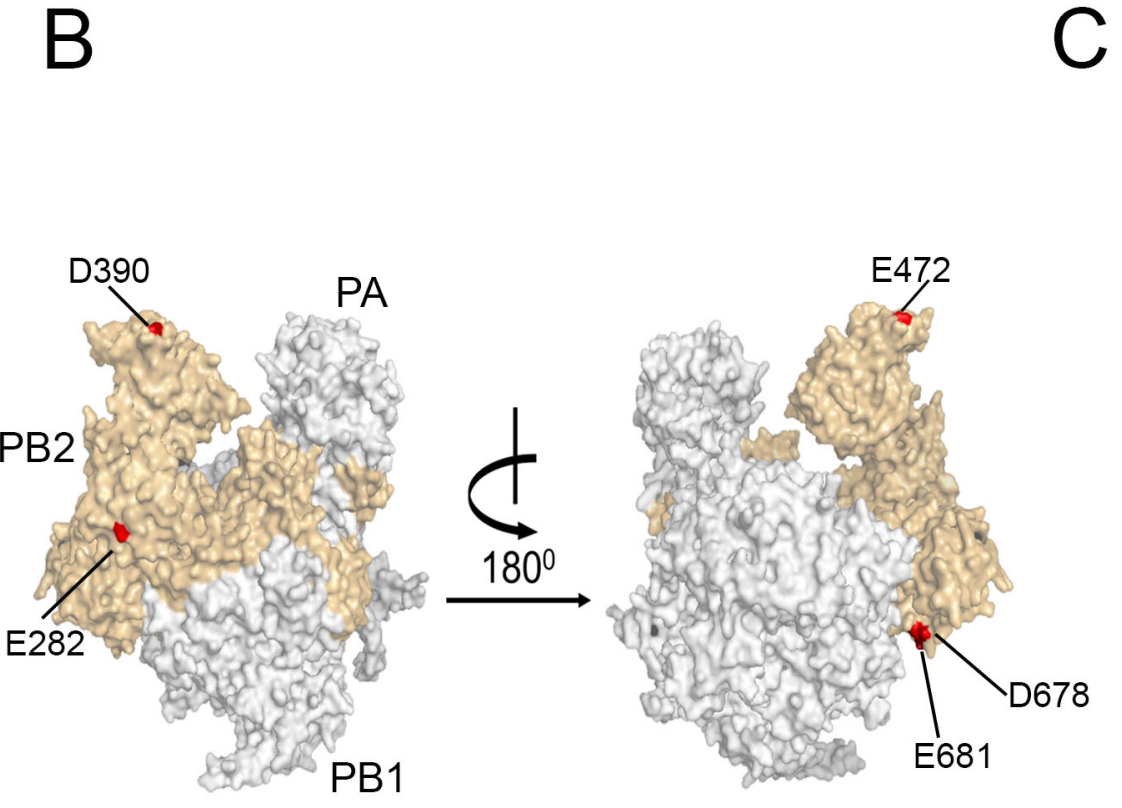

Heterotrimeric polymerase

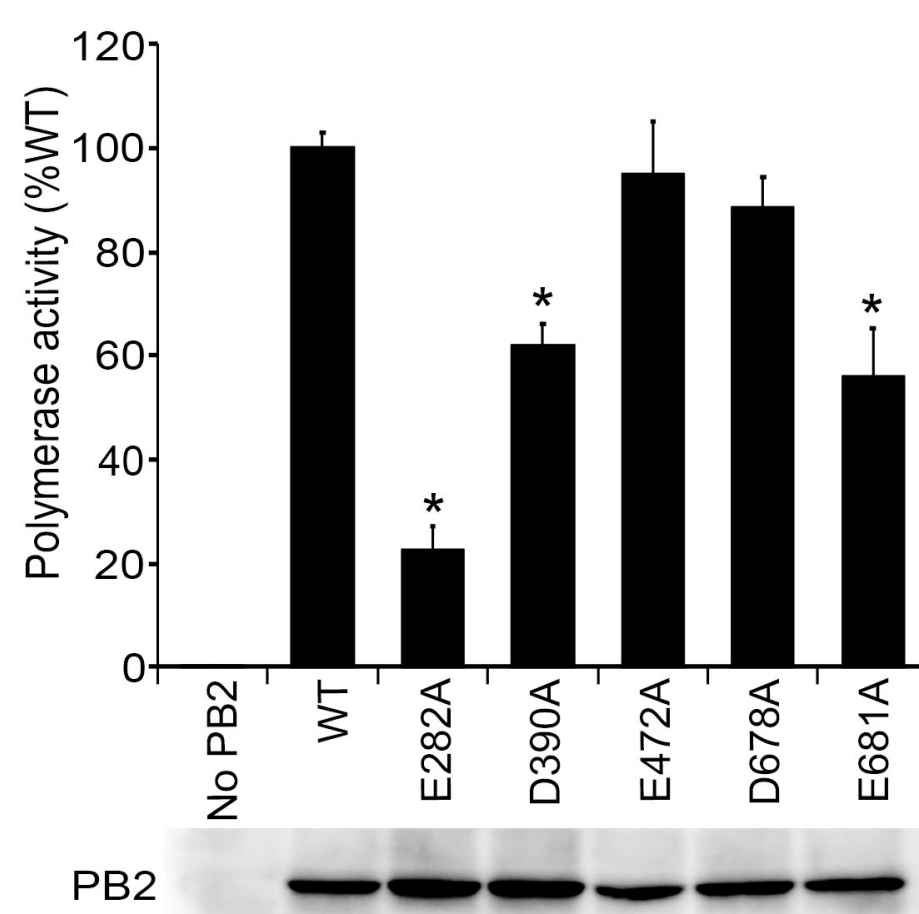


A

B
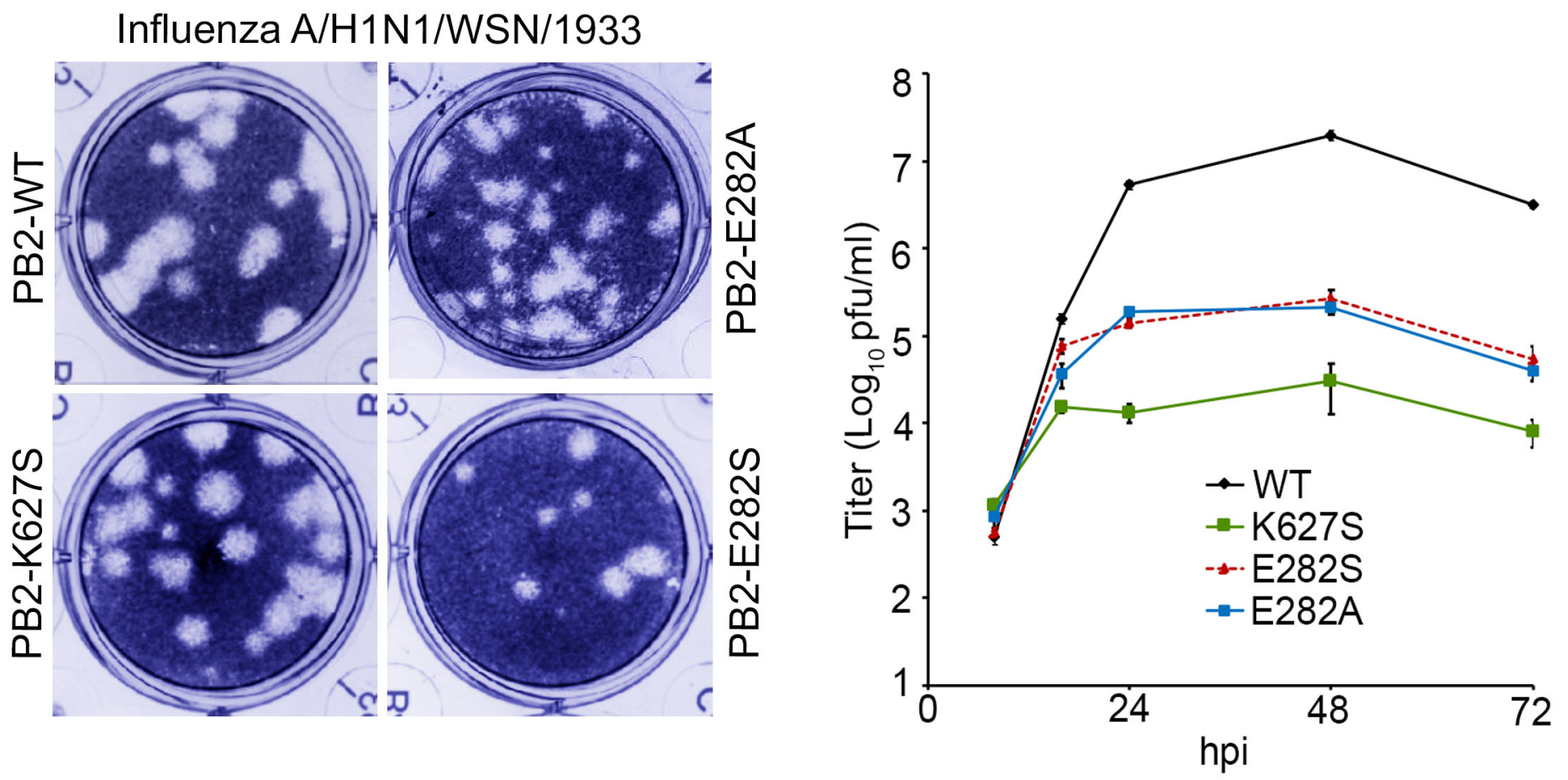

6

$D$
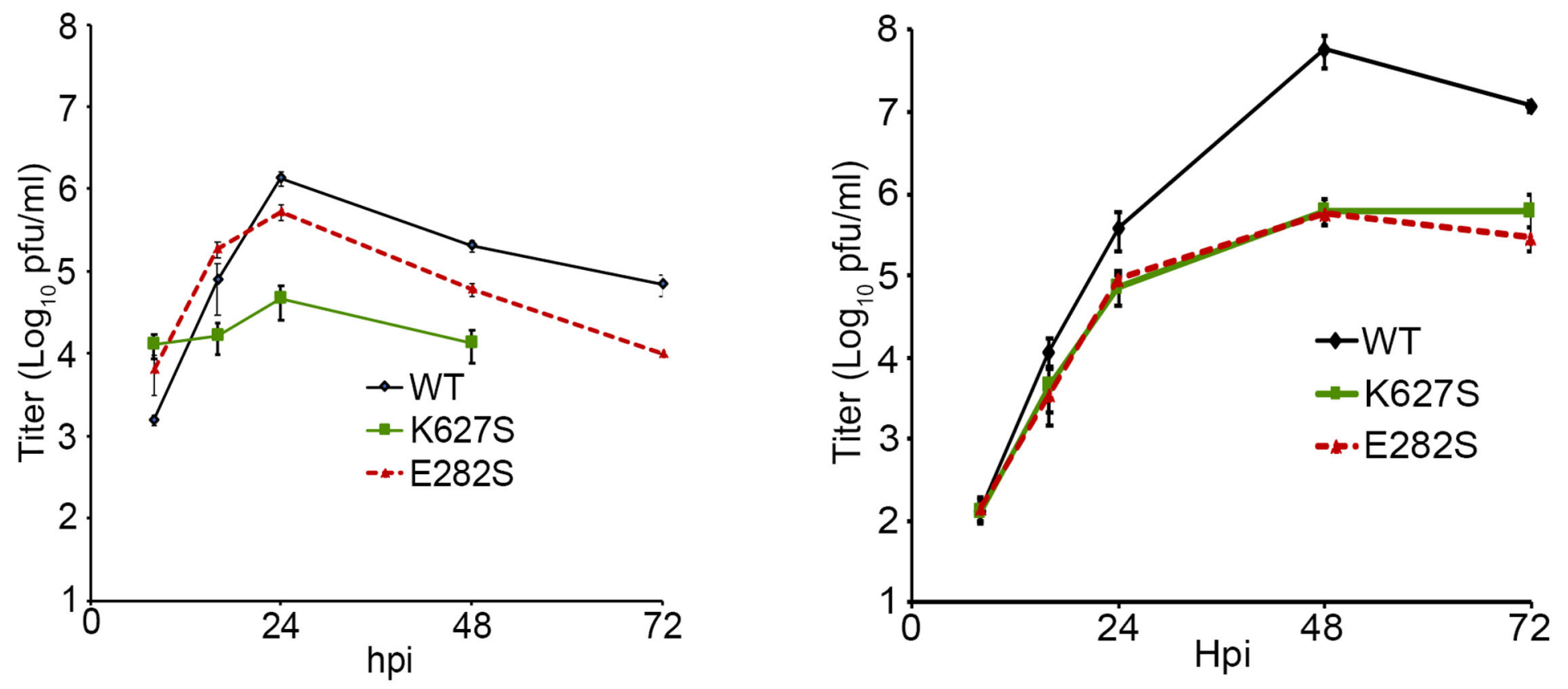
A

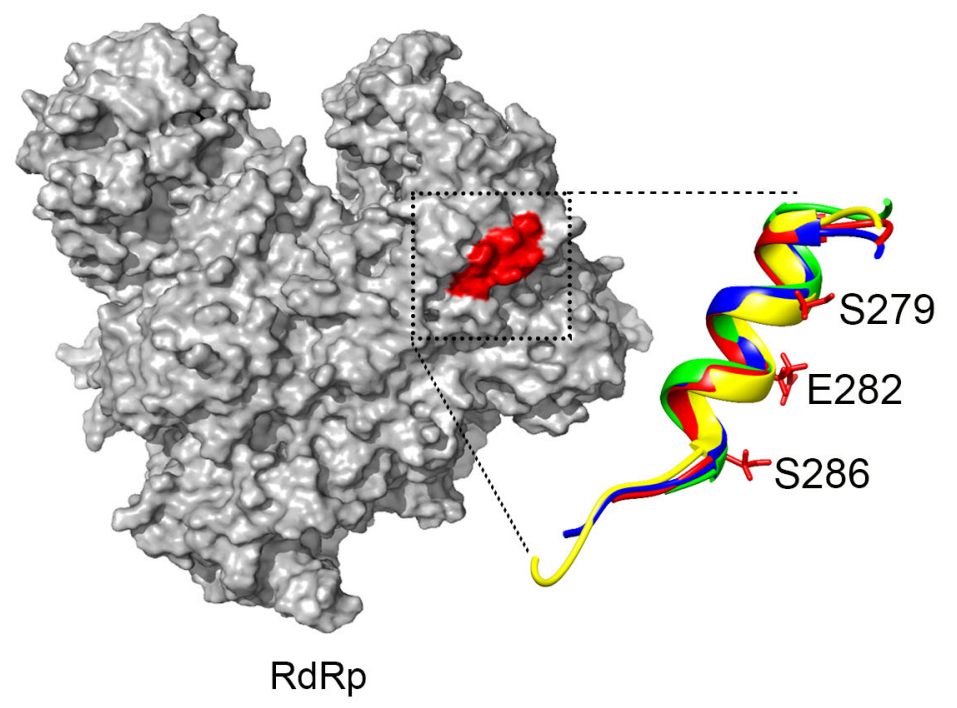

C
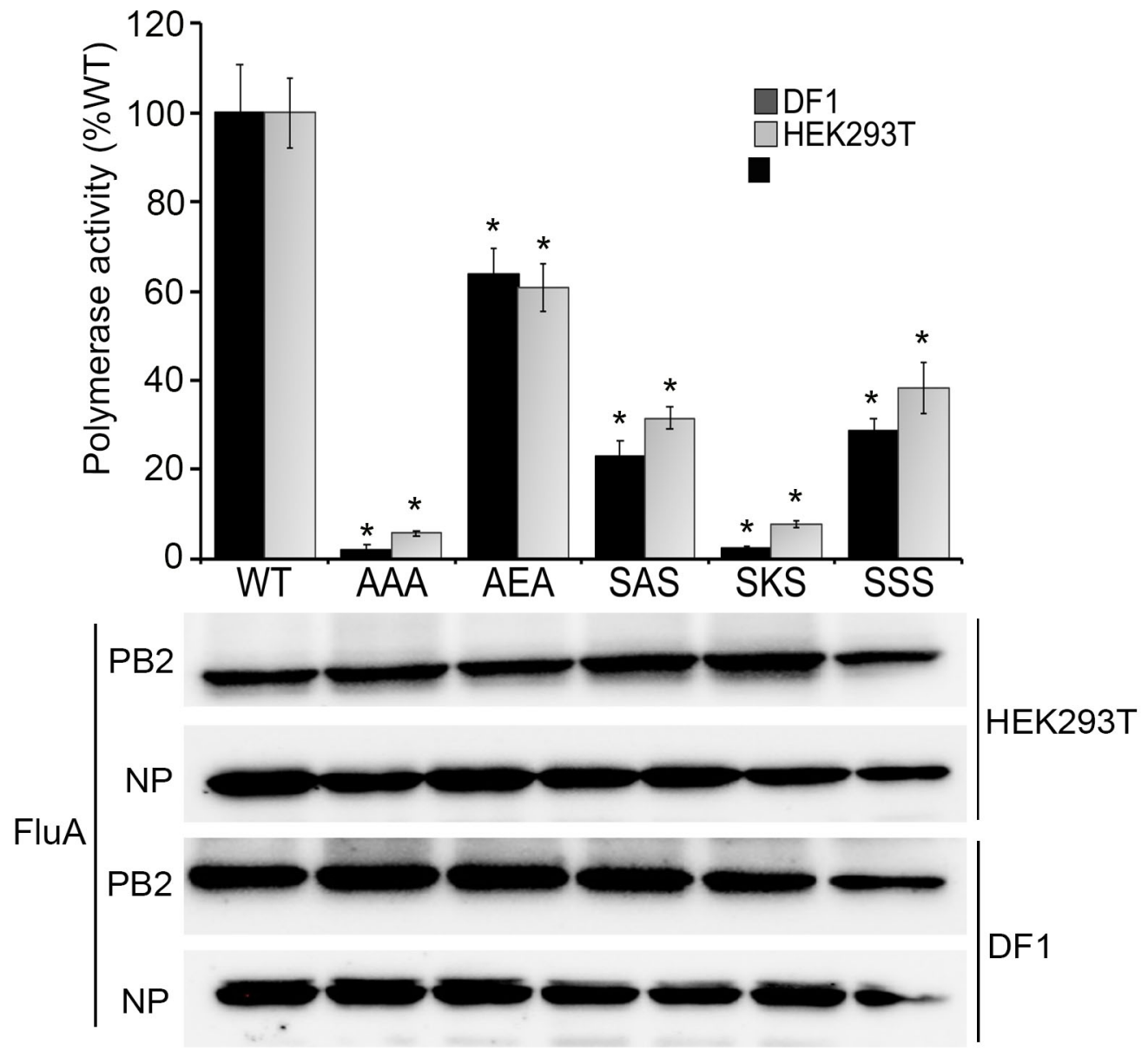

Flu A/H17N10: DPLASL L SMCHTT Flu A/H1N1: DPLASL L EMCHST Flu C: MNAKSKLVEY I KST Flu D: F TPAEK I RDY I SSV Flu B: SNPLELAVE I ANKT

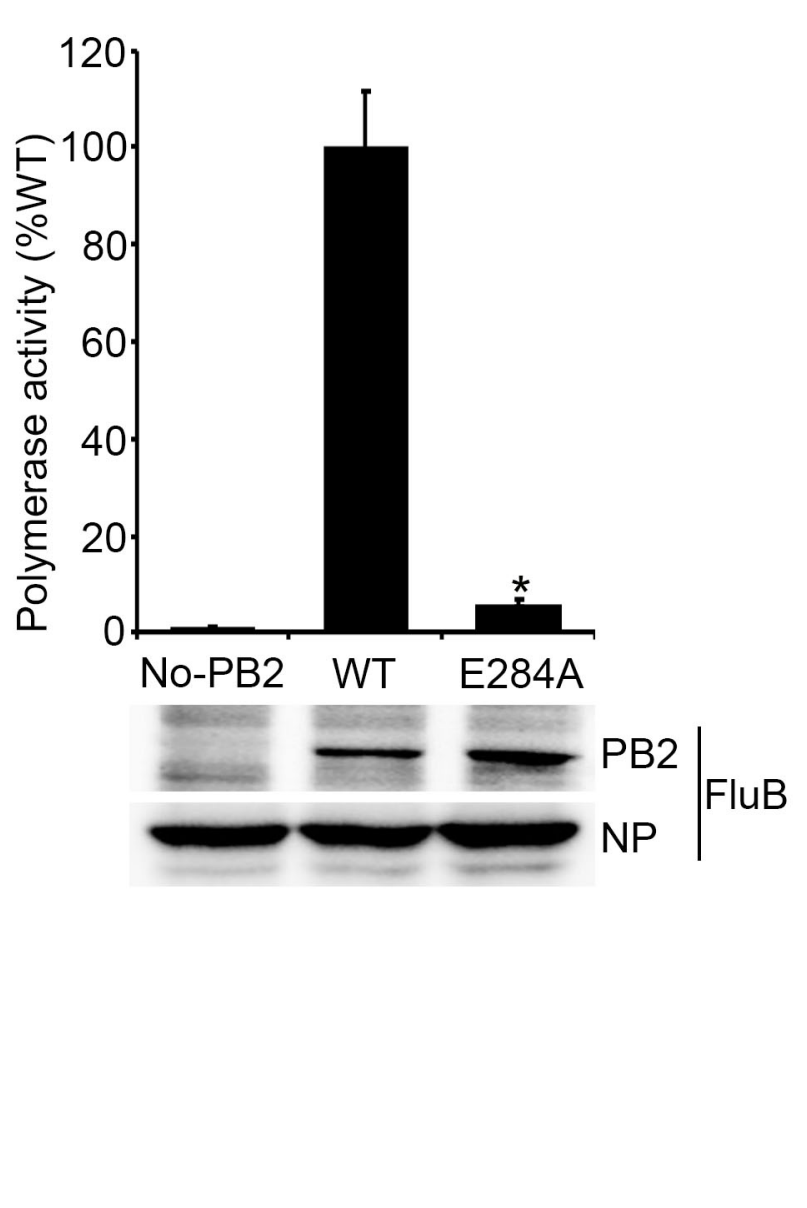


bioRxiv preprindoi: https://doi.org/10.1101/2021.04.12.439585; this v@Rßß3osted April 13, 2021. The copyright holder for this preprint (which A (as not certified by peer caview) is allowed without permission.

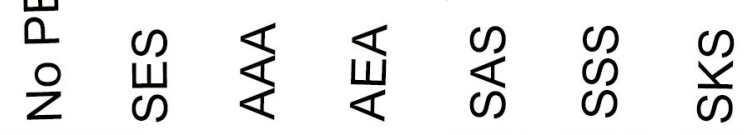

IP:PB2

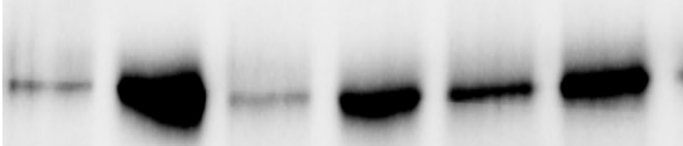

RNP (NP)

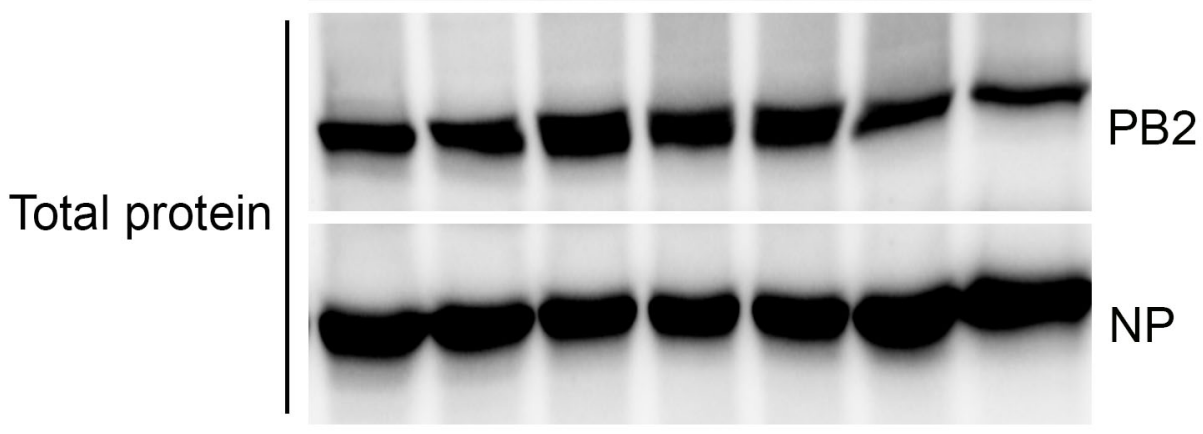

B

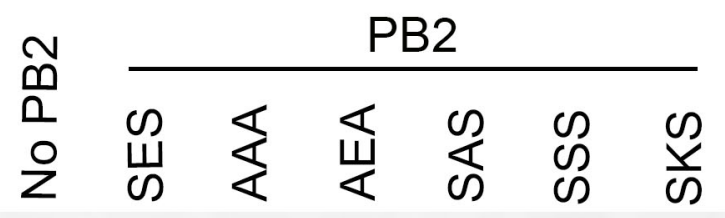

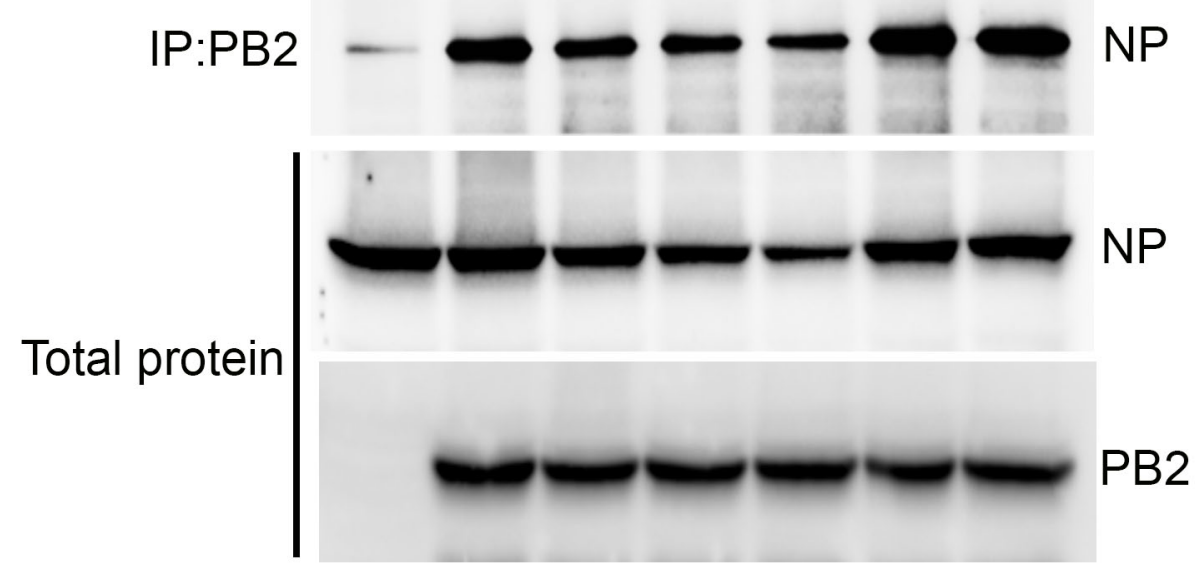

C

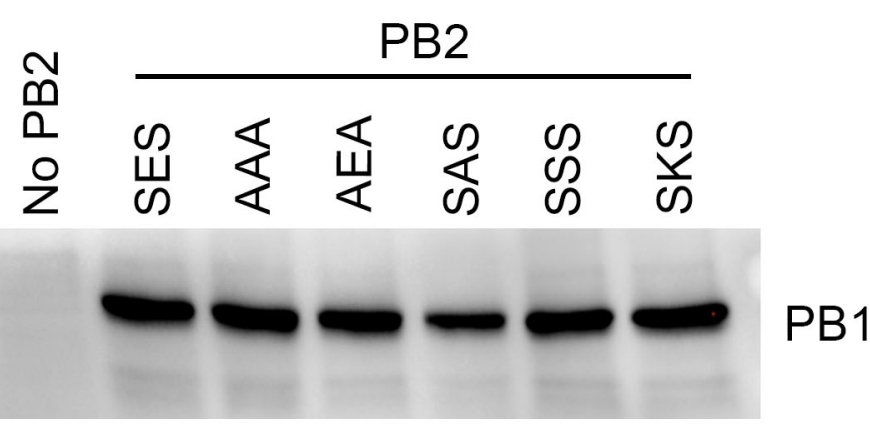

Total protein

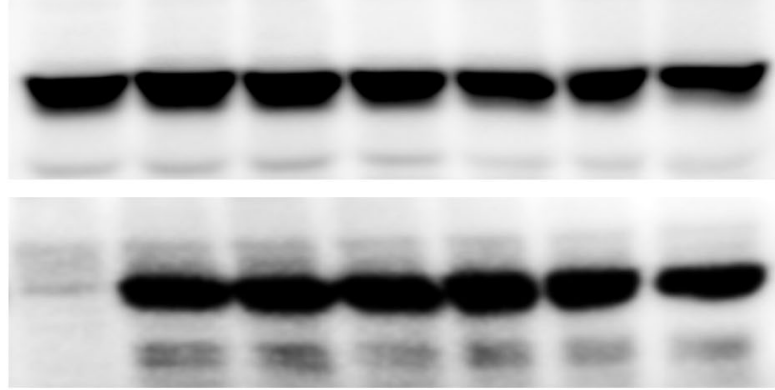

PB1 
bioRxiv preprint doi: https://doi.org/10.1101/2021.04.12.439585; this version posted April 13, 2021. The copyright holder for this preprint (which was not certified by peer review) is the author/funder. All rights reserved. No reuse allowed without permission.

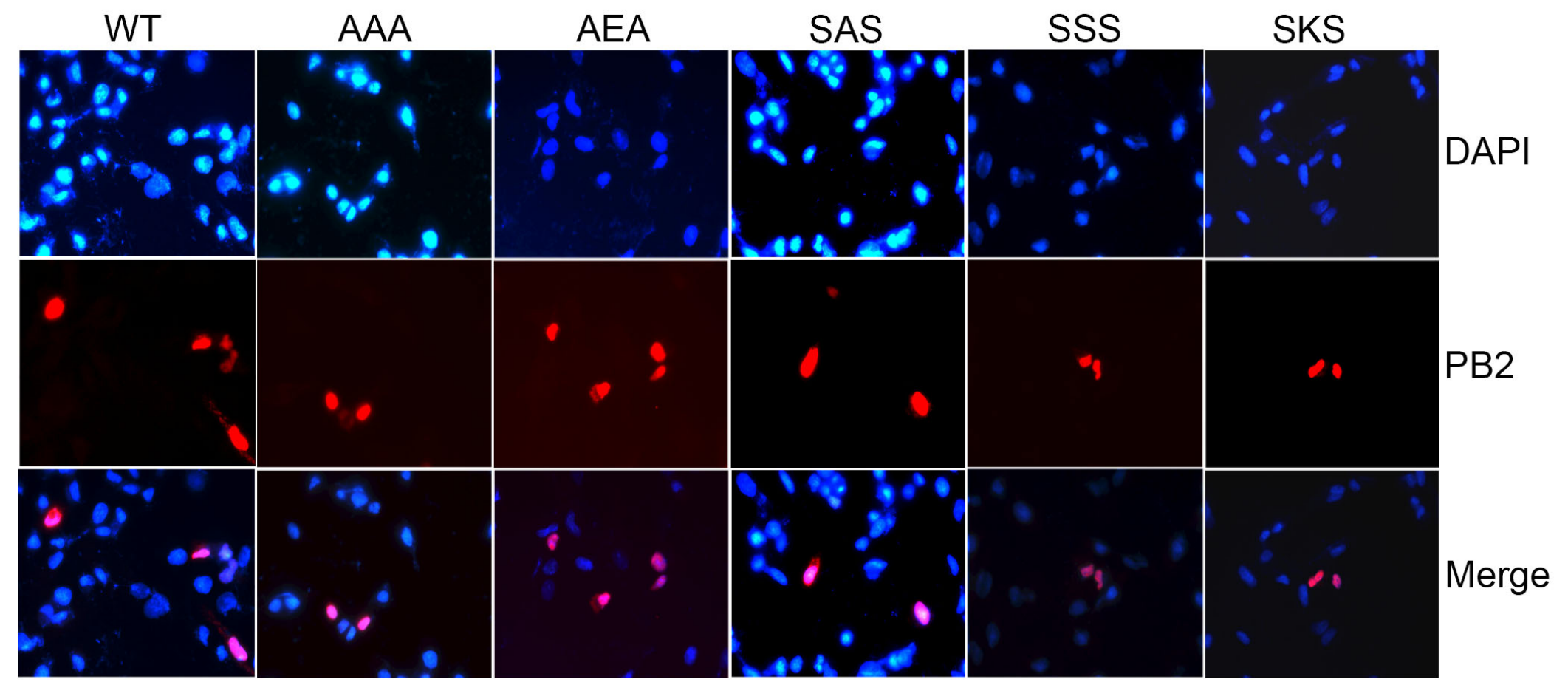


A

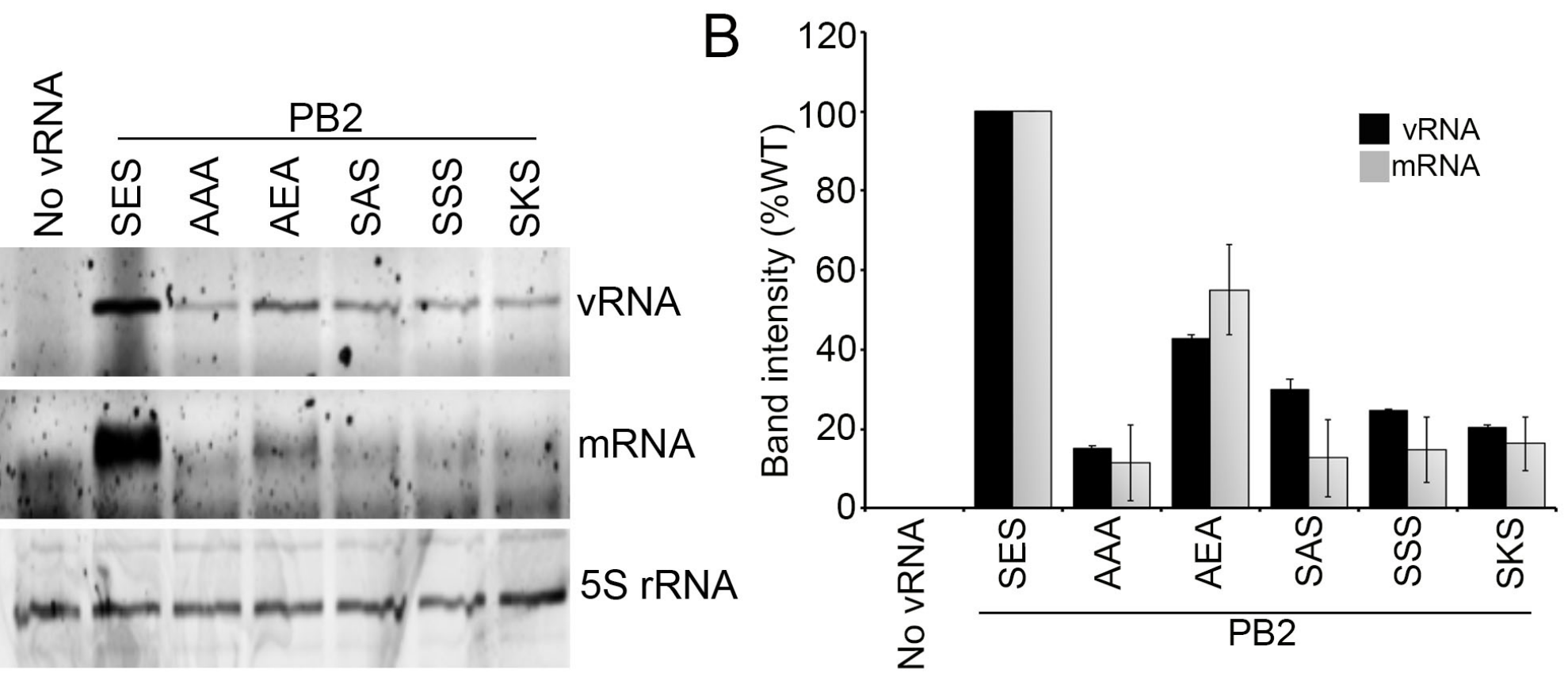


